\title{
Identification of kinetin riboside as a repressor of CCND1 and CCND2 with preclinical antimyeloma activity
}

\author{
Rodger E. Tiedemann, ${ }^{1}$ Xinliang Mao, ${ }^{2,3}$ Chang-Xin Shi, ${ }^{1}$ Yuan Xiao Zhu, ${ }^{1}$ Stephen E. Palmer, \\ Michael Sebag, ${ }^{1}$ Ron Marler, ${ }^{1}$ Marta Chesi, ${ }^{1}$ Rafael Fonseca, ${ }^{1}$ P. Leif Bergsagel, ${ }^{1}$ \\ Aaron D. Schimmer, ${ }^{2,3,4}$ and A. Keith Stewart ${ }^{1}$

\begin{abstract}
${ }^{1}$ Mayo Clinic, Comprehensive Cancer Center, Division of Hematology and Oncology, Scottsdale, Arizona, USA. ${ }^{2}$ Princess Margaret Hospital and Ontario Cancer Institute, Toronto, Ontario, Canada. ${ }^{3}$ McLaughlin Centre for Molecular Medicine, Toronto, Ontario, Canada.
\end{abstract} \\ ${ }^{4}$ Sinai-McLaughlin Assay and Robotic Technologies Facility, Mount Sinai Hospital, Toronto, Ontario, Canada.
}

\begin{abstract}
Knockout and transgenic studies in mice demonstrate that normal somatic tissues redundantly express 3 cyclin $\mathrm{D}$ proteins, whereas tumor cells seem dependent on a single overexpressed cyclin $\mathrm{D}$. Thus, selective suppression of the individual cyclin $\mathrm{D}$ deregulated in a tumor represents a biologically valid approach to targeted cancer therapy. In multiple myeloma, overexpression of 1 of the cyclin $D$ proteins is a ubiquitous feature, unifying at least 7 different initiating genetic events. We demonstrate here that RNAi of genes encoding cyclin D1 and cyclin D2 (CCND1 and CCND2, respectively) inhibits proliferation and is progressively cytotoxic in human myeloma cells. By screening a chemical library using a cell-based assay for inhibition of CCND2 trans-activation, we identified the plant cytokinin kinetin riboside as an inhibitor of CCND2 trans-activation. Kinetin riboside induced marked suppression of CCND2 transcription and rapidly suppressed cyclin D1 and D2 protein expression in primary myeloma cells and tumor lines, causing cell-cycle arrest, tumor cell-selective apoptosis, and inhibition of myeloma growth in xenografted mice. Mechanistically, kinetin riboside upregulated expression of transcription repressor isoforms of cAMP-response element modulator (CREM) and blocked both trans-activation of CCND2 by various myeloma oncogenes and cis-activation of translocated CCND1, suggesting induction of an overriding repressor activity that blocks multiple oncogenic pathways targeting cyclin $D$ genes. These data support targeted repression of cyclin $\mathrm{D}$ genes as a therapeutic strategy for human malignancies.
\end{abstract}

\section{Introduction}

Multiple myeloma (MM) is a postgerminal center B cell malignancy characterized by clonal plasma cell expansion. The disease manifests clinically with anemia, monoclonal immunoglobulin, renal insufficiency, and lytic bone lesions and currently affects 63,000 people in the United States alone (1); moreover, as it is currently incurable, MM causes a disproportionate $2 \%$ of all cancer deaths.

Although myeloma tumors present with complex karyotypes and an assortment of structural and numerical chromosomal abnormalities, these tumors are unified in their ubiquitous targeting of cyclin D genes for overexpression. Of MM tumors, $54 \%$ overexpress cyclin D1 (CCND1), 48\% overexpress cyclin D2 (CCND2), 3\% overexpress cyclin D3 (CCND3), and 8\% overexpress both CCND1 and CCND2 (2). Deregulated expression of single CCND genes in MM occurs as the result of selective cyclin $\mathrm{D}$ gene trans-activation by deregulated transcription factors or from translocation of a single

Nonstandard abbreviations used: AP-1, activator protein 1 ; BACH2, BTB and CNC homology 1 basic leucine zipper transcription factor 2; BAFF, TNF (ligand) superfamily member 13b; CCND1, human cyclin D1 gene; CDK, cyclin-dependent kinase; CRE, cAMP-response element(s); CREM, CRE modulator; FGFR3, FGF receptor 3; HMCL, human myeloma cell line(s); ICER, inducible cAMP early repressor, an isoform of CREM; IGH, immunoglobulin heavy-chain locus; MAF, human v-maf musculoaponeurotic fibrosarcoma oncogene homolog (avian); MARE, MAF response element; MM, multiple myeloma; MMSET, multiple myeloma SET domain-containing protein; MTS, 3-(4,5-dimethyl-2-yl)-5-(3-carboxymethoxyphenyl)-2-(4-sulfophenyl)2H-tetrazolium; MTT, 3-[4,5-dimethylthiazol-2-yl]-2,5-diphenyl tetrazolium bromide; NT, nontargeting; PP2A, protein phosphatase $2 \mathrm{~A}$; RSV, Rous sarcoma virus.

Conflict of interest: The authors have declared that no conflict of interest exists. Citation for this article: J. Clin. Invest. 118:1750-1764 (2008). doi:10.1172/JCI34149. cyclin D gene to a strong enhancer locus. Notably, nearly half of all myeloma tumors possess 1 of 5 recurrent 14q32 immunoglobulin heavy-chain $(I G H)$ enhancer translocations that directly dysregulate CCND1 or CCND3 or that dysregulate FGF receptor 3 (FGFR3) or human v-maf musculoaponeurotic fibrosarcoma oncogene homolog (avian) (MAF) family transcription factors, which ultimately trans-activate CCND2 (3): 11q13 (CCND1); 6p21 (CCND3); 4p16 (FGFR3/multiple myeloma SET domain-containing protein [MMSET]); 16q23 (MAF); or 20q12 (v-maf musculoaponeurotic fibrosarcoma oncogene homolog B [avian] [MAFB]). The remaining myeloma tumors are hyperdiploid and have multiple trisomies involving chromosomes 3, 5, 7, 9, 11, 15, 19, and 21. Although hyperdiploid MM tumors generally lack recurrent 14q32 translocations, they too show overexpression of a cyclin $\mathrm{D}$ with biallelic trans-activation of CCND1 or CCND2 (2).

$\mathrm{D}$ cyclins are essential regulators of blood-cell division. By modulating cyclin-dependent kinase (CDK) activity, cyclin D proteins regulate retinoblastoma protein phosphorylation, acting as a switch to commit the cell to a program of DNA replication and cell division. While knockout mouse models indicate that most somatic tissues can develop in the complete absence of D cyclins (4) and even CDK4/6 $(5,6)$, the hematopoietic compartment in particular is dependent upon these proteins for proliferation and cell survival (4).

Normal proliferating human tissues typically coexpress 3 cyclin D proteins (D1, D2, and D3) that are essentially interchangeable $(7,8)$ due to substantive functional redundancy $(9)$. In contrast, many hematopoietic cancers such as MM achieve sustained cell- 

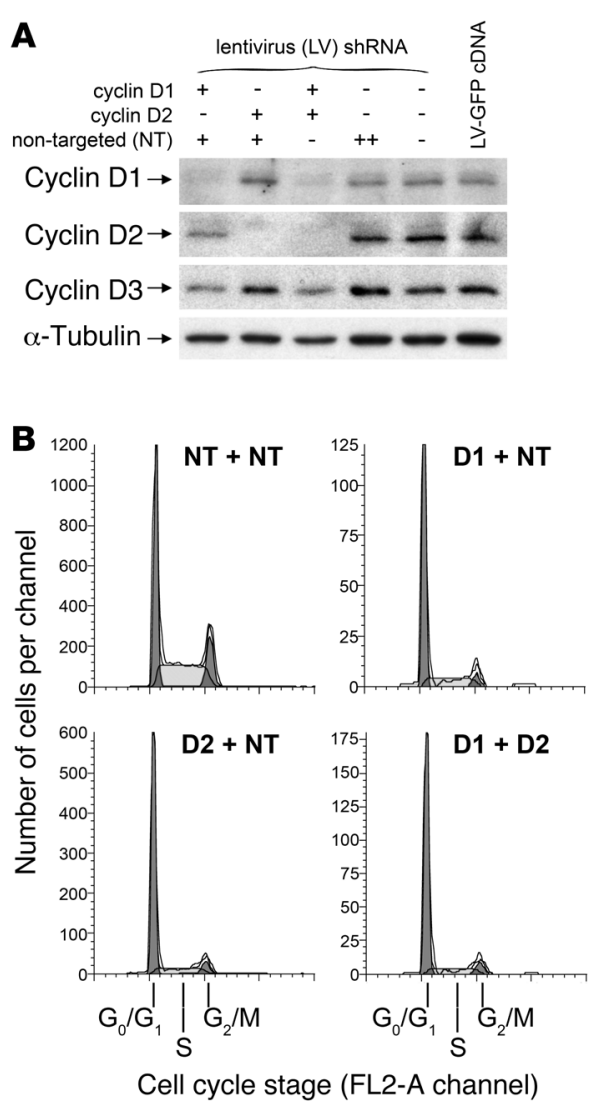
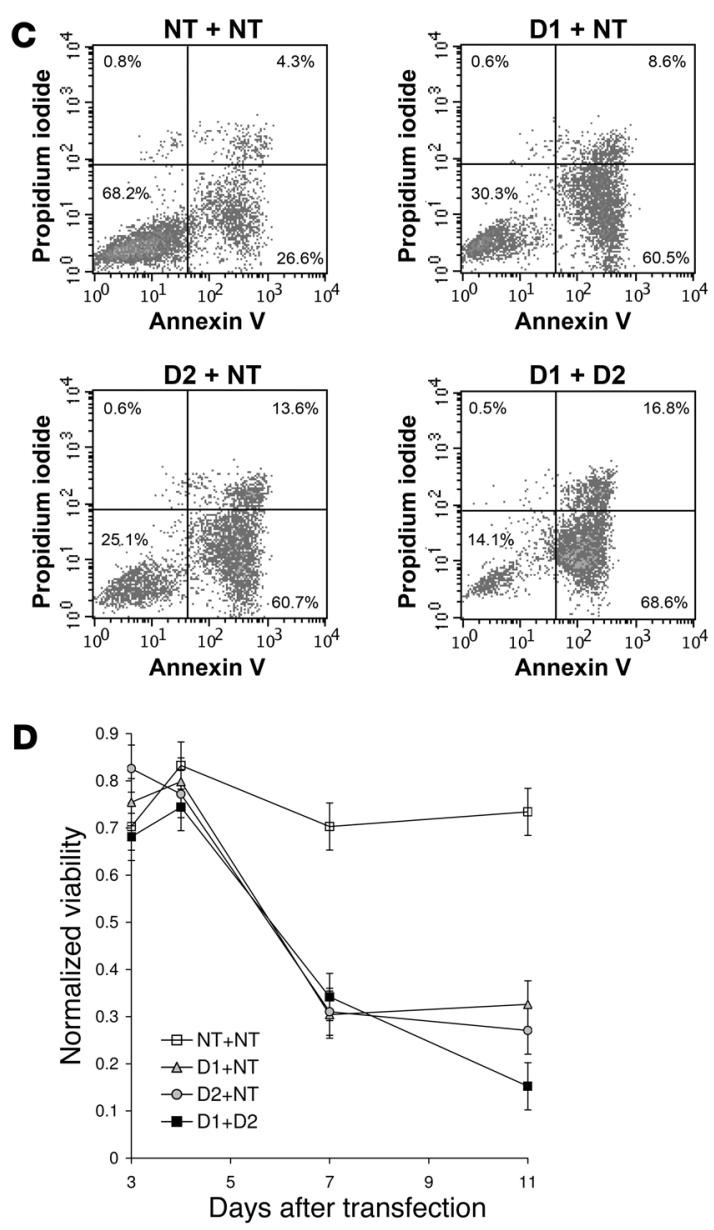

Figure 1

Lentiviral shRNA suppression of cyclin D1 and D2 induces cell-cycle arrest and delayed apoptosis in H929 tumor cells. (A) Immunoblot analysis of cyclin D1, D2, and D3 expression in H929 myeloma cells 65 hours after infection with lentiviruses expressing cyclin D1 or cyclin D2 shRNA, NT control shRNA, or GFP cDNA. $\alpha$-Tubulin levels are shown as loading controls. (B) Cell-cycle profiles of H929 cultures 4 days after lentivirus infection, comparing the effects of suppression of cyclin D1 or D2 with control NT lentivirus infection and with lentivirus-induced suppression of both cyclin D1 and cyclin D2. To ensure equivalent exposure to lentivirus and shRNA, cells infected with both D1 lentivirus and D2 lentivirus (D1 + D2) are compared with cells infected with D1 lentivirus and NT lentivirus (D1 + NT), D2 lentivirus and NT lentivirus (D2 + NT), or 2 aliquots of NT lentivirus (NT + NT). (C) H929 apoptosis was examined serially by flow cytometry (in this example on day 11) after cyclin D suppression. Viable cells (left lower quadrants) are distinguished from early apoptotic (annexin V-positive) or late apoptotic (propidium iodide-positive) cells. (D) Time course of H929 viability after infection with D1 + NT, D2 + NT, D1 + D2, or control NT + NT lentiviruses. Viability was determined by annexin $\mathrm{V}$ and propidium iodide staining, as illustrated in $\mathbf{C}$, and is normalized to uninfected $\mathrm{H} 929 ; 5 \%$ error in viability is shown.

cycle deregulation by overexpressing a single cyclin D. MM tumors thus differ from normal proliferating tissues in their dependence upon a single cyclin D for excess growth. Therefore, selective suppression of the cyclin $\mathrm{D}$ gene exploited by such tumors may be an effective and universal targeted therapeutic strategy.

While CDK inhibitors are currently being tested with some success in clinical trials for cancer, we believe that inhibition of individual cyclin Ds may offer similar or greater therapeutic specificity in MM and comparable malignancies because at a molecular level these tumors differ both qualitatively and quantitatively from normal tissues in their expression of D cyclins but are less distinguished by their expression of CDK. In this paper, we identify kinetin riboside as what we believe is a novel inhibitor of cis- and trans-activation of CCND1 and CCND2 and explore its potential as a therapeutic agent for MM.

\section{Results}

Silencing CCND1 and CCND2 induces cell-cycle arrest and cytotoxicity in myeloma cells. As myeloma tumors universally dysregulate a cyclin D gene, commonly CCND1 or CCND2, we tested to determine whether silencing of 1 or both of these genes could induce growth arrest or cytotoxicity in myeloma cells and thus whether targeting of specific cyclin Ds could represent a valid therapeutic approach in this malignancy. H929 myeloma cells were infected with lentivirus vectors expressing interfering shRNA targeting either cyclin D1 or cyclin D2 or with control lentivirus expressing nontargeting (NT) shRNA, and the effects on cellular proliferation and viability were determined (Figure 1, Table 1, and Supplemental Table 1; supplemental material available online with this article; doi:10.1172/JCI34149DS1). Using conditions that resulted in more than $99 \%$ cellular infection ( $\mathrm{R}$. Tiedemann, unpublished 


\section{Table 1}

Cell-cycle profile of H929 myeloma cells 4 days following lentiviral suppression of cyclin D1 and D2

$\begin{array}{lcccc} & \mathbf{G}_{\mathbf{0}} / \mathbf{G}_{\mathbf{1}} & \mathbf{S} & \mathbf{G}_{\mathbf{2}} / \mathbf{M} & \text { Normalized } \Delta \mathbf{S} \\ \text { Lentivirus/shRNA } & & & & \\ \text { No LV } & 46.5 & 38.7 & 14.8 & 0.0 \\ \text { NT + NT } & 53.8 & 34.6 & 11.4 & -10.6 \\ \text { D1 + NT } & 75.7 & 19.4 & 4.9 & -49.9 \\ \text { D2 + NT } & 76.9 & 16.7 & 6.4 & -56.8 \\ \text { D1 + D2 } & 79.9 & 13.9 & 6.1 & -64.1\end{array}$

H929 cells were infected with various combinations of lentiviruses expressing NT shRNA or interfering shRNA targeting cyclin D1 or D2 expression, as described in Figure 1. For each condition, the percentage of cells in $G_{0} / G_{1}, S$, or $G_{2} / M$ phases and the decrease in S-phase entry $(\Delta S)$ relative to uninfected cells is shown.

observations) and effective cyclin D gene silencing (Figure 1A), we observed substantial $\mathrm{G}_{0} / \mathrm{G}_{1}$ cell-cycle arrest with silencing of either cyclin D1 or cyclin D2 (Figure 1B) but not with control NT lentivirus, confirming the importance of cyclin $\mathrm{D}$ dysregulation to H929 cells. Suppression of both cyclin D1 and D2 caused a 64\% reduction in S-phase progression (Table 1). Significantly, silencing of cyclin D1 and/or D2 was also associated with delayed myeloma cell apoptosis (Figure 1C) that was evident by day 7 after infection (Figure 1D). Combined cell-cycle arrest and apoptosis together resulted in substantial reductions in viable H929 numbers. Overall, these results, together with previous demonstrations of the viability of cyclin D1- or D2-deficient mice (10), suggest that specific silencing of cyclin D2 or D1 could be employed to achieve a targeted antimyeloma therapeutic effect.

High-throughput assay for inhibitors of MAF-driven CCND2 trans-activation. As cyclin D2 suppression was inhibitory to $\mathrm{H} 929$ and as poor prognosis myeloma variants commonly dysregulate CCND2 (2), we sought to discover pharmaceutical inhibitors of CCND2 trans-activation. To identify inhibitors, we developed a highthroughput chemical genetics screen using NIH3T3 reporter cells stably overexpressing the CCND2-LUC promoter-reporter construct. $3 \mathrm{~T} 3$ cells were chosen for their adherence, which is useful for high-throughput screening. To ensure that trans-activation of the cyclin D2 promoter in this system was relevant to myeloma, the 3T3 cells were cotransfected with MAF-expressing retrovirus. MAF directly trans-activates CCND2 and is recurrently overexpressed in $\mathrm{t}(14 ; 16)$ myeloma (11). In our system, basal cyclin D2 promoter activity was readily detectable and was upregulated by MAF coexpression (Supplemental Figure 1). To eliminate discovery of nonspecific cytotoxic agents that indirectly suppress CCND2 or LUC by causing $3 \mathrm{~T} 3$ cytotoxicity, a 3-(4,5-dimethyl-2-yl)-5-(3-carboxymethoxyphenyl)-2-(4-sulfophenyl)-2H-tetrazolium (MTS) viability assay was performed in parallel with our primary CCND2-LUC assay. Additionally, to determine the MAF dependence or independence of CCND2 inhibitors identified during primary screening, inhibitors were retested in a secondary screen both in the presence and absence of the MAF vector. Thus, together our primary and secondary screens could identify and distinguish drugs that caused MAFdependent or -independent suppression of CCND2 transcription and could eliminate drugs that caused only indirect suppression of CCND2 or LUC secondary to $3 \mathrm{~T} 3$ cytotoxicity.
In an optimized and automated assay, $3 \mathrm{~T} 3$ reporter cells were plated in 96-well plates by robot and treated for 16 hours at $37^{\circ} \mathrm{C}$ with compounds at final concentrations of approximately $5 \mu \mathrm{M}$ with less than $0.1 \%$ DMSO. In control wells, cells were treated with buffer alone. More than 4,000 compounds from LOPAC $(n=1,280)$, Prestwick $(n=1,120)$, and Spectrum $(n=2,000)$ libraries were screened. Compounds that induced greater than 2 -fold reduction in CCND2 promoter-expressed LUC relative to viability were assigned the status of putative "hits." The assay coefficient of variance $(\mathrm{CV})$ during screening was less than $10 \%$.

Surprisingly, the majority of hits from the LOPAC and Prestwick libraries were glucocorticoid family members. From screening the Spectrum compound library (Figure 2A), we identified 38 hits, of which 29 were also glucocorticosteroids. The mechanisms by which corticosteroids decrease cyclin $\mathrm{D}$ gene expression has been the subject of parallel investigation and is described elsewhere (12). Notably, 9 nonsteroid compounds were identified as putative suppressors of CCND2; these included kinetin riboside, dihydrogambogic acid, monensin, patulin, gentian violet, pararosaniline pamoate, pristimerin, aklavine, and camptothecin.

Exclusion of nonspecific assay bits and prioritization of putative CCND2 transcription inhibitors. Drugs that decreased LUC but not viability in the screening assay may have acted to reduce trans-activation of the cyclin D2 promoter or alternatively may have directly inhibited the LUC enzyme. To distinguish between these possibilities, assay hits were retested against 3T3 cells that overexpress LUC under the control of an Rous sarcoma virus (RSV) promoter. From this testing, we discovered that patulin nonspecifically reduced LUC activity and both patulin and aklavine caused excessive 3T3 cytotoxicity (X. Mao and R. Tiedemann, unpublished observations). Therefore, these drugs were excluded from further analysis. However, other hits, including kinetin riboside (Figure 2B), did not decrease LUC when driven by the control RSV promoter and therefore act via the CCND2 promoter.

To focus our subsequent efforts, we next excluded compounds with reported toxicities likely to prevent clinical systemic use, including pararosaniline pamoate and gentian violet $(13,14)$. Monensin was also excluded after experiments showed excessive nonspecific toxicity toward nonmalignant hematopoietic cells (R. Tiedemann, unpublished observation). Similarly, camptothecin was excluded as it directly interferes with DNA synthesis and likely modulates CCND2 expression only indirectly by its S-phase activity $(15,16)$.

To assess whether the remaining putative CCND2 inhibitors identified from screening act via MAF or are MAF-independent, these compounds were reassessed against reporter $3 \mathrm{~T} 3$ cells both in the presence and absence of retrovirally expressed MAF. Untransformed 3T3 cells do not have detectable levels of MAF by immunoblotting (R. Tiedemann and X. Mao, unpublished observations). Each of the 3 compounds, including kinetin riboside, pristimerin, and dihydrogambogic acid, suppressed the CCND2 promoter both in the absence and presence of MAF (results for kinetin riboside shown in Figure 2B). We concluded that these compounds function independently of MAF to inhibit CCND2 trans-activation. However, since triterpenoids and dihydrogambogic acid have previously been described as showing anticancer activity and as recent studies suggest that these likely suppress cyclin D secondarily via modulation of NF-kB $(17,18)$, we focused our detailed studies on kinetin riboside.

Kinetin riboside inbibits CCND1 plus CCND2 trans-activation and prevents cell-cycle entry from $G_{0} / G_{1}$. Kinetin riboside (N6-furfury- 

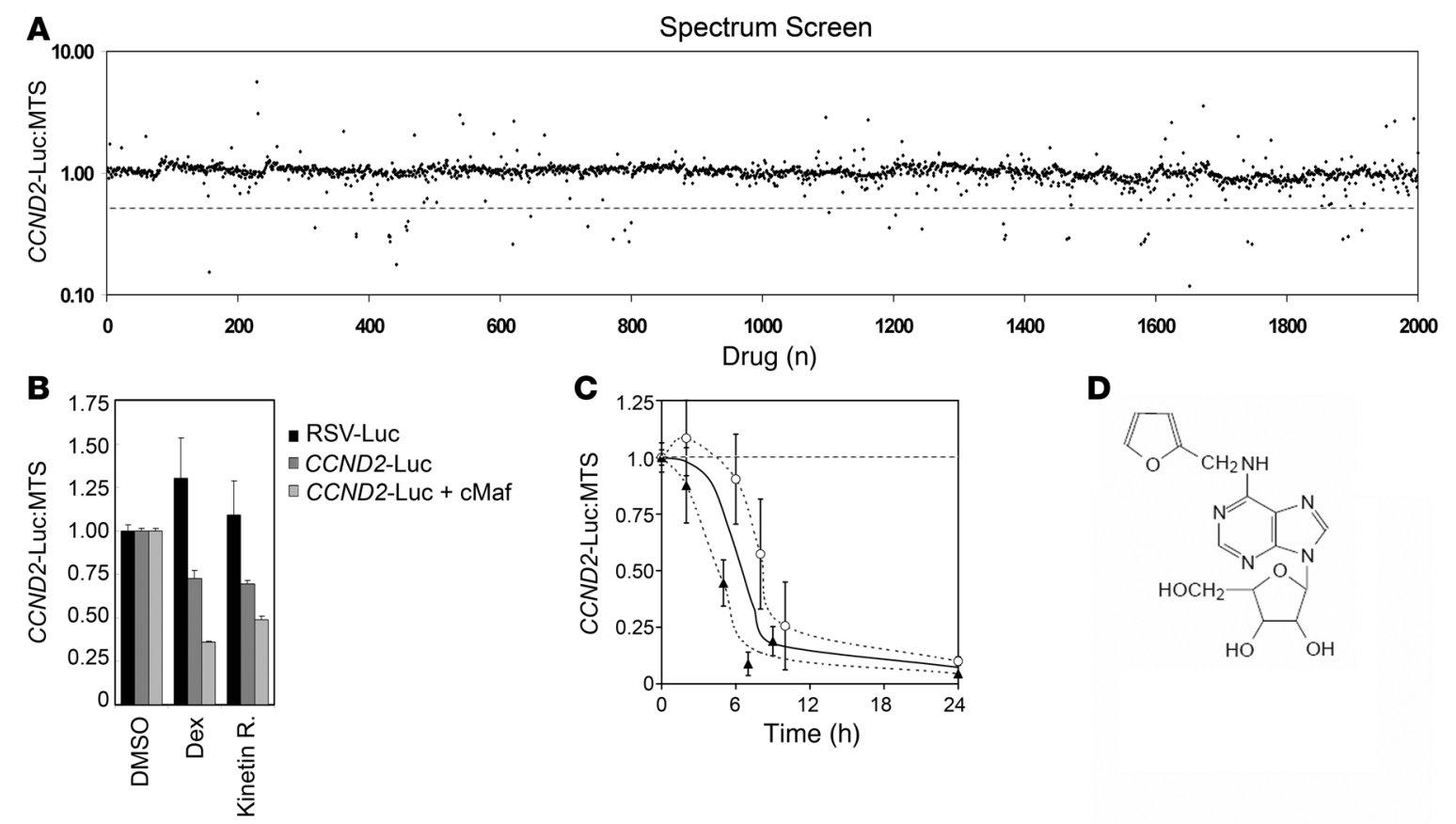

D

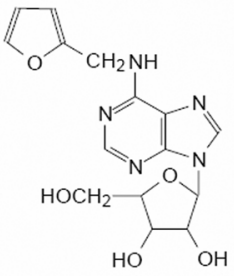

Figure 2

Identification of kinetin riboside by drug library screening for inhibitors of CCND2 promoter trans-activation in an NIH3T3 cell reporter system. (A) Compounds were screened for inhibition of CCND2 promoter trans-activation at 16 hours in a 3T3 reporter model in which the MAF-transactivating factor was coexpressed with the CCND2 promoter-driving LUC expression. The results of screening the Spectrum library is shown as a dot plot comparing each compound's assay position ( $x$ axis) with its effect on MAF-driven CCND2 promoter trans-activation (measured by LUC) relative to effects on 3 T3 viability (measured by MTS; $y$ axis). Compounds below the dotted line were defined as putative hits. LOPAC and Prestwick drug libraries were also screened but are not shown. (B) Repeat testing of kinetin riboside (kinetin R), dexamethasone, and vehicle against reporter cells expressing LUC driven by a control RSV promoter or the CCND2 promoter, with and without MAF coexpression, showing that LUC suppression by these drugs is mediated specifically by the CCND2 promoter. Repression of CCND2 promoter activity by kinetin riboside is not restricted to trans-activation induced by MAF. (C) Suppression of CCND2-driven LUC protein levels in 3T3 following kinetin riboside $(10 \mu \mathrm{M})$ treatment. Results of separate experiments are plotted (triangles and circles); each point is the mean of duplicates \pm SEM. The overall curve of best fit is depicted as a solid line. Despite an estimated LUC half-life of 3 hours, kinetin riboside induces approximately $80 \%$ suppression by 9 hours, suggesting that suppression of the CCND2 promoter begins within 1-4 hours. (D) Chemical structure of kinetin riboside.

ladenosine) reproducibly induced rapid suppression of the CCND2 promoter in $3 \mathrm{~T} 3$ fibroblasts (Figure 2C) and is a synthetic cytokinin and nucleoside derivative. Cytokinins are a family of natural plant hormones that stimulate quiescent plant cells to undergo cell division $(19,20)$ via transcriptional induction of cyclin D3 (21); therefore, kinetin riboside-induced suppression of CCND2 in our assay suggested this compound may have related but antagonistic properties on cyclin D transcription in mammalian cells.

To further investigate kinetin riboside, we first validated the influence of this compound on cyclin D gene expression in a human malignancy model. Kinetin riboside was tested against a panel of human myeloma cell lines (HMCL). These MM cell lines have a range of transforming events relevant to myeloma and, as a consequence, all overexpress cyclin D1 or D2. As shown in Figure $3 \mathrm{~A}$, kinetin riboside caused significant suppression of cyclin D1 and/or D2 in HMCL but did not suppress cyclin D3. Thus, kinetin riboside induced suppression of cyclin D2 in H929 and KMS11 cells despite the presence in these HMCL of FGFR3 and MAF translocations that cause biallelic trans-activation of CCND2 (2). Surprisingly, kinetin riboside also induced suppression of cyclin D1 in U266 cells despite cis deregulation of CCND1 in this HMCL resulting from chromosomal relocation of the IGH enhancer to CCND1. Suppression of both cis- and trans-activated CCND1 or
CCND2 in HMCL and inhibition of direct MAF-driven CCND2 promoter trans-activation in $3 \mathrm{~T} 3$ cells suggest kinetin riboside may inhibit cyclin $\mathrm{D}$ gene expression via recruitment of a transcriptional repressor (see below).

HMCL proliferate ex vivo and are not always faithfully representative of primary MM tumor cells. We therefore tested the effect of kinetin riboside on cyclin D expression in purified CD $138^{+}$ cells from MM patient bone marrow aspirates. In 4 of 5 cases (Figure 3B), kinetin riboside induced suppression of the major cyclin D protein expressed in primary myeloma cells beyond any decline associated with in vitro culture. In a fifth case (tumor E), derived from a plasma cell leukemia patient, cyclin D1 was highly expressed after culture and was unaffected by kinetin riboside. The time course of kinetin riboside activity was next assessed in H929 cells (Figure 3C). Kinetin riboside caused suppression of cyclin D1 and D2 proteins within 6 hours; however, cyclin D3 was not significantly affected until later, when a proportion of cells were likely to be undergoing apoptosis (see below).

To verify that the suppression of cyclin D1 and D2 induced by kinetin riboside is a direct effect and does not occur secondary to cellular arrest in S-phase when CCND1 and CCND2 are normally downregulated (22), we examined the effects of kinetin riboside on the cell cycle of HMCL cultured in standard media 

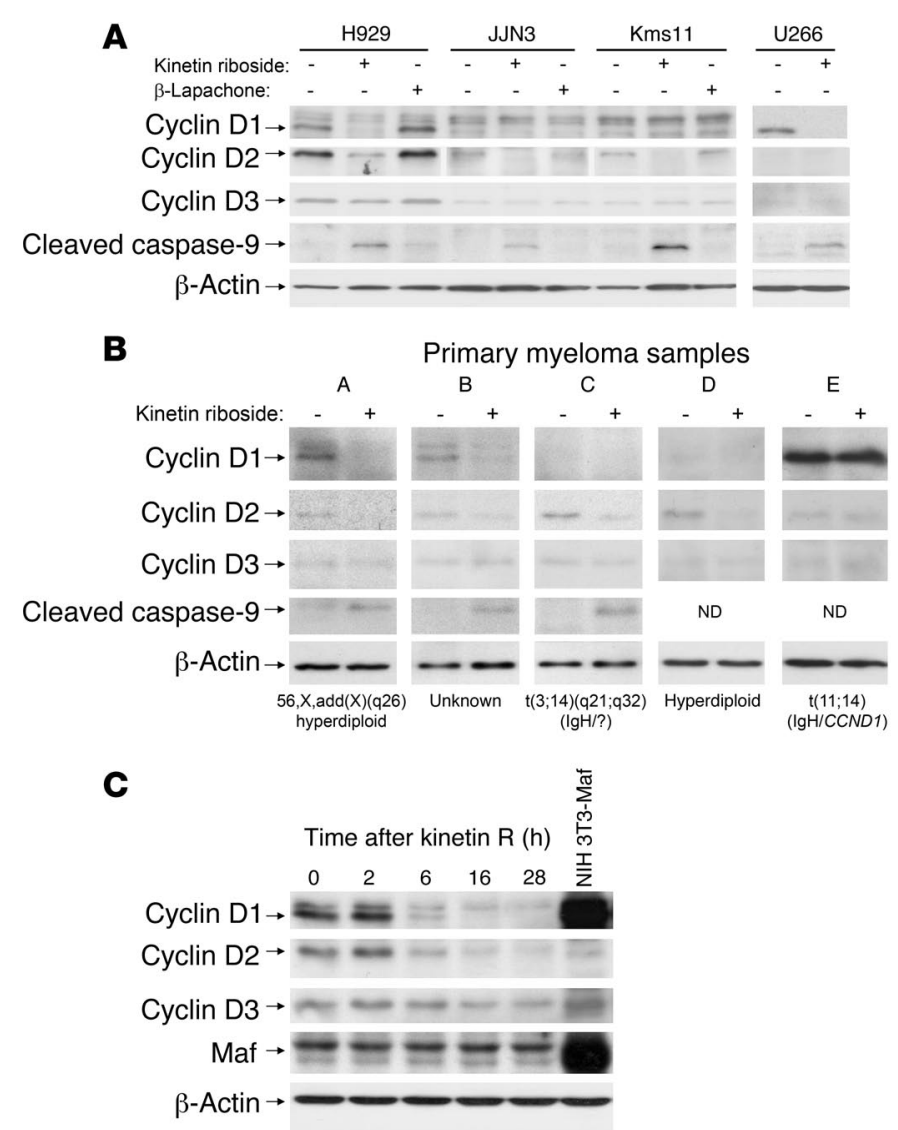

(Figure 4 and Table 2). Within 20 hours, kinetin riboside caused the fraction of cells entering S-phase to fall by $50 \%-70 \%$ in all HMCL tested and caused a corresponding increase in $G_{0} / G_{1}$ cells. This suggests that rather than causing indirect suppression of cyclin D as a result of first arresting cells in S-phase (as can be demonstrated with conventional S-phase cytotoxic agents; R. Tiedemann, unpublished observations), kinetin riboside acts to directly suppress cyclin D1 $\pm \mathrm{D} 2$. Secondary cell-cycle arrest at $\mathrm{G}_{0} / \mathrm{G}_{1}$ is consistent with cyclin D suppression.

Kinetin riboside inhibits tumor cell growth and induces selective apoptosis of primary MM cells in mixed-lineage bone marrow culture. Kinetin riboside is inhibitory to myeloma cell lines with $\mathrm{IC}_{50}<1.7 \mathrm{mg} / \mathrm{l}(5 \mu \mathrm{M})$ in 5/12 HMCL and $<5 \mathrm{mg} / \mathrm{l}(15 \mu \mathrm{M})$ in $11 / 12 \mathrm{HMCL}$ at 72 hours and remains active when HMCL are treated with a cocktail of MM growth cytokines including IL-6, IGF-1, and TNF ligand superfamily member $13 \mathrm{~b}$ (BAFF) (Figure 5, A-C, and Table 3). Kinetin riboside is also inhibitory to the mantle cell lymphoma line GRANTA and the cyclin D-expressing Burkitt lymphoma line RAMOS with $\mathrm{IC}_{50}<1.6 \mathrm{mg} / \mathrm{l}(<4.7 \mu \mathrm{M})$. Similarly, kinetin riboside is inhibitory to a spectrum of breast, pancreatic, melanoma, and glioma tumor lines, frequently at $\mathrm{IC}_{50}$ concentrations below or equal to those observed for myeloma. Dysregulation of cyclin D genes, most commonly CCND1, has previously been described in these tumors.

In $\mathrm{MM}$, kinetin riboside-induced cellular inhibition is largely mediated by apoptosis as HMCL and primary MM cells treated with kinetin riboside showed caspase- 9 cleavage by 16 hours (see Figure 3 ) and acquisition of annexin $\mathrm{V}$ binding by 72 hours (Figure 5, B and D). 3-[4,5-dimetylthiazol-2-yl]-2,5-diphenyl tetrazolium

\section{Figure 3}

Kinetin riboside causes suppression of cyclins D1 and D2 and caspase activation in human MM. (A) Immunoblot showing suppression of cyclins D1 and D2, but not D3, and induction of caspase-9 cleavage by kinetin riboside $(10 \mu \mathrm{M})$ at 16 hours in HMCL. Kinetin riboside effects are compared with those of an unrelated control cytotoxic $\beta$-lapachone $\left(1 \mu \mathrm{M}\right.$, approximately $\left.4 \times \mathrm{IC}_{50}\right)$ or with DMSO vehicle. For visualization, the JJN3 cyclin D2 blot was exposed approximately 2-fold longer than cyclin D2 blots for H929, KMS11, and U266. (B) Kinetin riboside $(10 \mu \mathrm{M})$ induces similar cyclin D1 and D2 suppression and caspase cleavage in CD138-purified primary myeloma cells at 16 hours, shown by immunoblotting. A plasma cell leukemia sample (patient E) was unaffected. The cytogenetics status of the tumor cells or IGH gene translocation is shown, if known. (C) Cyclin D and MAF protein levels in $\mathrm{H} 929$ cells after exposure to kinetin riboside $(10 \mu \mathrm{M})$, showing suppression of cyclin D1 and D2 within 6 hours.

bromide (MTT) assay responses in U266 largely reflect cell-cycle arrest, however, as little or no apoptosis was observed in kinetin riboside-treated $\mathrm{U} 266$ cells by flow cytometry (for annexin V binding), even by 96 hours (Figure 5B). Notably MM tumor cells became CD138 negative in response to kinetin riboside treatment; loss of surface CD138 during plasma cell apoptosis is well established (23) and is associated with annexin $V$ positivity following kinetin riboside treatment (see Supplemental Figure 2).

As mouse embryos that lack D cyclins eventually die from hematopoietic failure (4), the bone marrow might be predicted to be sensitive to drugs targeting cyclin $\mathrm{D}$, particularly if all $3 \mathrm{D}$ cyclins are inhibited. We therefore treated bone marrow samples from MM patients with kinetin riboside to assess the relative cytotoxicity of this drug on myeloma cells and nonmalignant marrow progenitors. Bone marrow samples were processed to remove nonnucleated red cells and then treated for 72 hours. Importantly, kinetin riboside preferentially killed primary CD138 ${ }^{+}$myeloma cells but was substantially less cytotoxic to nonmalignant marrow cells (Figure 5, D and E, and Supplemental Figures 2 and 3). By comparison, the conventional antimyeloma cytotoxic, melphalan, killed more healthy CD138- marrow cells than malignant myeloma cells in the same assay (R. Tiedemann, unpublished observations).

Synergistic cytotoxicity of kinetin riboside and glucocorticosteroids. Glucocorticosteroids such as dexamethasone represent one of the most effective treatments available in myeloma. Moreover, corticosteroids repeatedly induced CCND2 promoter suppression in our screening assay; therefore we tested the combination of kinetin riboside and dexamethasone for additive cytotoxicity against HMCL. Significantly, combining kinetin riboside and dexamethasone (at $\mathrm{IC}_{50}$ or lower concentrations) induced greater than additive cytotoxicity (Figure 5C). Thus, treatment of JJN3 or MM.1S with kinetin riboside and dexamethasone together resulted in enhanced cytotoxicity that overcame the relative insensitivities of these HMCL to low-dose dexamethasone or to kinetin riboside, respectively. Similarly, the cell line MY5 was only partially responsive to low doses of either agent alone; however, the combination resulted in marked cytotoxicity. In all 3 cases, the cytotoxicity resulting from the combination of agents was greater than the sum of the cytotoxicities of each agent when used alone.

Kinetin riboside suppresses cyclin D1 and D2 expression from full-length endogenous CCND1 and CCND2 promoters in myeloma cells. As we have shown, kinetin riboside induces rapid suppression of a truncated 


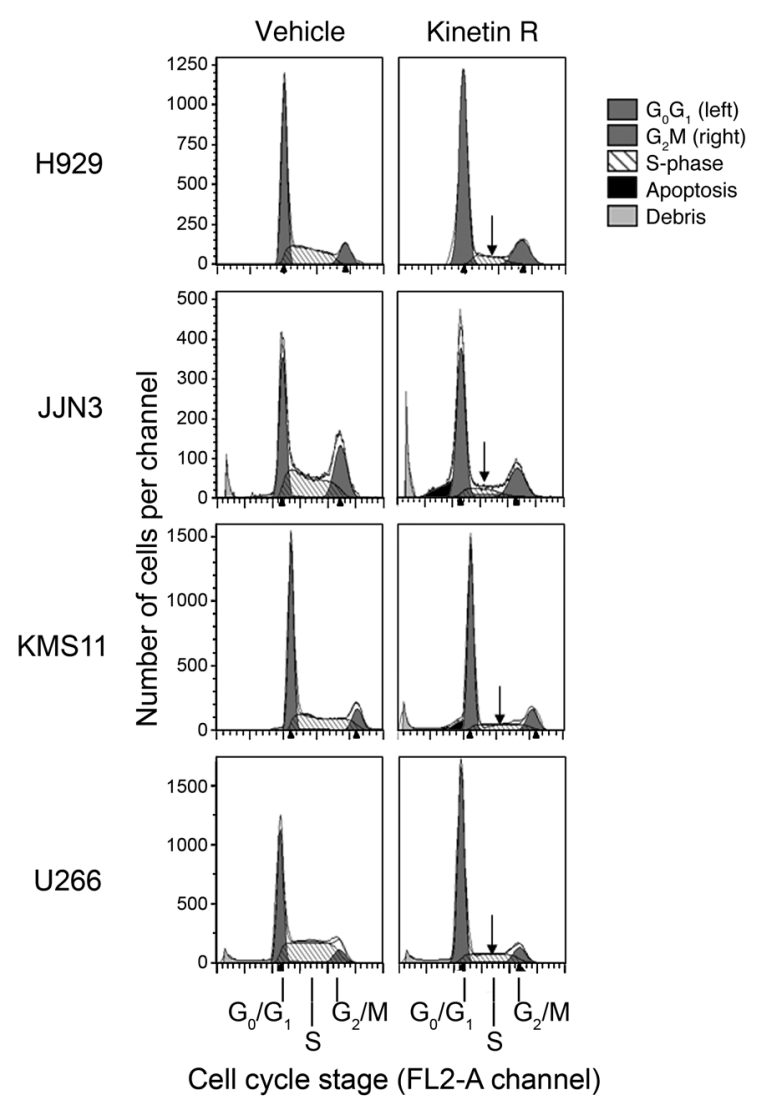

Figure 4

Kinetin riboside inhibits cell-cycle transit from $\mathrm{G}_{0} / \mathrm{G}_{1}$ to $\mathrm{S}$-phase. Cell-cycle profile of HMCL treated with DMSO vehicle or kinetin riboside $(10 \mu \mathrm{M})$ at 20 hours. Left and right peaks represent $\mathrm{G}_{0} / \mathrm{G}_{1}$ and $G_{2} / M$ phases, respectively. Similar results were obtained with duplicate experiments.

human CCND2 promoter in 3T3 cells and also induces rapid suppression of cyclin D1 and D2 proteins in human myeloma cells. However, plausibly, cyclin D protein suppression in myeloma cells could be attributed to off-target toxicity associated with posttranscriptional cyclin D effects rather than being due to direct effects on the cyclin D1 and D2 promoters. Therefore, to determine whether kinetin riboside induces on-target suppression of full-length endogenous cyclin D1 and D2 promoters in MM cells or whether instead kinetin riboside causes posttranscriptional cyclin D degradation, we next engineered H929 and KMS11 myeloma cells to express cyclin D1 or D2 from a heterologous CMV promoter and then reexamined the influence of kinetin riboside on cyclin $\mathrm{D}$ expression (Figure 6A). Notably, whereas cyclin D2 expressed from the endogenous CCND2 promoter in KMS11 cells was suppressed by kinetin riboside, cyclin D2 expressed from a heterologous CMV promoter in KMS11 was not. Similarly, while no endogenous cyclin D1 was detectable in KMS11 cells, ectopic expression of cyclin D1 in KMS11 from the CMV promoter was again not subject to kinetin riboside suppression. Furthermore, while cyclin D1 expressed from the endogenous CCND1 promoter in H929 cells was detectable and was suppressed by kinetin riboside, cyclin D1 expressed from the heterologous CMV promoter in H929 cells was not suppressed by kinetin riboside. Therefore, in MM cells, modu- lation of cyclin D1 and D2 protein levels by kinetin riboside, like modulation of CCND2-LUC activity in 3T3 cells, is mediated solely by on-target effects on the CCND1 or CCND2 promoters, and suppression of these proteins is not a consequence of unrelated nonspecific cytotoxicity or off-target posttranslational mechanisms.

Expression of cyclin D2 and D1 from a heterologous promoter rescues KMS11 myeloma cells from kinetin riboside. Although kinetin riboside induces rapid suppression of CCND1 and CCND2 via their endogenous promoters, it is plausible that the subsequent effects of kinetin riboside on tumor cell division and viability may be unrelated to cyclin D1 or D2 effects, which might instead represent bystander phenomena. To address this possibility, we next evaluated the cell-cycle and apoptosis profiles of kinetin riboside-treated KMS11 MM cells expressing cyclin D1 or D2 from heterologous CMV promoters (Figure 6, B-E). As shown earlier, kinetin riboside induces $\mathrm{G}_{0} \mathrm{G}_{1}$-phase cell-cycle arrest in myeloma cells, putatively from cyclin $D$ suppression. In a repeat experiment, $G_{0} G_{1}$ arrest was again observed in control KMS11 cells treated with kinetin riboside for 20 hours, while no $\mathrm{G}_{0} \mathrm{G}_{1}$ arrest developed in kinetin riboside-treated KMS11 cells that expressed cyclin D1 or D2 (or both) from a heterologous CMV promoter (Figure 6, B and C). These data strongly indicate that the primary mechanism of kinetin riboside-induced proliferative arrest in myeloma cells is modulation of cyclin D gene expression and that this effect may be overcome by reexpression of cyclin D.

We next examined the role of cyclin D suppression and cellcycle arrest by kinetin riboside on KMS11 myeloma cell viability (Figure 6, D and E). KMS11 cells infected with control adenovirus for 5 days and treated with DMSO vehicle for 3 days showed mild (<20\%) virus-related cytotoxicity, proportional to GFP expression (Figure 6D; cells strongly positive for GFP are increasingly annexin $\mathrm{V}$ positive). However, the majority of adenovirus-infected cells expressed remained viable. Notably, kinetin riboside treatment caused the number of viable (annexin V negative) KMS11 cells infected with control adenovirus to decrease by more than $50 \%$ (Figure 6E) and caused a corresponding increase in the proportion of annexin V-positive GFP-positive cells, consistent with an induction of apoptosis matching observed in this cell line in the absence of virus infection (Figure 5B). Significantly, however, kinetin riboside had no cytotoxic effect at 3 days on KMS11 cells expressing cyclin D2 from a heterologous CMV promoter and had less effect on KMS11 expressing cyclin D1 from a CMV promoter, indicating that the cytotoxicity of this compound in KMS11 relates to its modulation of cyclin D2 and, to a lesser extent, its modulation of cyclin D1. KMS11 cells normally express considerably more endogenous cyclin D2 than cyclin D1 - as a result of $t(4 ; 14)$ and $t(14 ; 16)$ translocations that upregulate FGFR3/MMSET and MAF, which

\section{Table 2}

Percentage of cells by cell-cycle phase and normalized change in S-phase compartment

\begin{tabular}{lccccccc}
\hline & \multicolumn{1}{c}{ Plus vehicle } & \multicolumn{1}{c}{ Plus kinetin riboside } & Normalized \\
HMCL & $\mathbf{G}_{\mathbf{0}} / \mathbf{G}_{1}$ & $\mathbf{S}$ & $\mathbf{G}_{2} / \mathbf{M}$ & $\mathbf{G}_{0} / \mathbf{G}_{1}$ & $\mathbf{S}$ & $\mathbf{G}_{2} / \mathbf{M}$ & $\Delta \mathbf{S}$ \\
H929 & 55 & 33 & 12 & 73 & 10 & 16 & $-69.7 \%$ \\
JJN3 & 37 & 38 & 25 & 62 & 15 & 23 & $-60.5 \%$ \\
KMS11 & 53 & 36 & 11 & 68 & 17 & 14 & $-52.8 \%$ \\
U266 & 42 & 50 & 8 & 68 & 21 & 10 & $-58.0 \%$ \\
\hline
\end{tabular}


Table 3

Kinetin riboside $\mathrm{IC}_{50}$ for myeloma (assayed $\pm \mathrm{IL}-6, \mathrm{IGF}-1$, and BAFF) and other tumor lines

\begin{tabular}{|c|c|c|c|c|}
\hline & $\begin{array}{c}\text { Medium } \\
\text { alone }(\mu \mathrm{M})\end{array}$ & $\begin{array}{c}\text { Medium } \\
\text { alone (mg/l) }\end{array}$ & $\begin{array}{l}+\mathrm{IL}-6 \text { + IGF-1 } \\
+ \text { BAFF }(\mu \mathrm{M})\end{array}$ & $\begin{array}{l}+\mathrm{IL}-6+\mathrm{IGF}-1 \\
+\mathrm{BAFF}(\mathrm{mg} / \mathrm{l})\end{array}$ \\
\hline \multicolumn{5}{|l|}{ Myeloma } \\
\hline $\mathrm{H} 929$ & 3 & 1.0 & 4 & 1.4 \\
\hline JJN3 & 8 & 2.8 & 9 & 3.1 \\
\hline KMS11 & 11 & 3.8 & 12.5 & 4.3 \\
\hline MM.1S & 12.9 & 4.5 & - & - \\
\hline MM.1R & 11 & 3.8 & - & - \\
\hline OCl-MY5 & 5.7 & 2.0 & - & - \\
\hline OPM1 & 6.1 & 2.1 & - & - \\
\hline OPM2 & 4.5 & 1.6 & 5.1 & 1.8 \\
\hline RPMI-8226 & 5 & 1.7 & 5 & 1.7 \\
\hline SKMM2 & 3 & 1.0 & 4 & 1.4 \\
\hline U266 & 20 & 6.9 & 20 & 6.9 \\
\hline UTMC2 & 3.3 & 1.1 & 6.1 & 2.1 \\
\hline \multicolumn{5}{|c|}{ Burkitt Iymphoma } \\
\hline RAMOS & 2.4 & 0.8 & - & - \\
\hline \multicolumn{5}{|c|}{ Mantle cell Iymphoma } \\
\hline GRANTA & 4.7 & 1.6 & - & - \\
\hline \multicolumn{5}{|c|}{ Breast carcinoma } \\
\hline MCF7 & 3.4 & 1.2 & - & - \\
\hline \multicolumn{5}{|c|}{ Pancreatic carcinoma } \\
\hline MIAPACA & 3.9 & 1.4 & - & - \\
\hline PANC & $>20$ & $>7$ & - & - \\
\hline \multicolumn{5}{|l|}{ Glioma } \\
\hline SF767 & 1.4 & 0.5 & - & - \\
\hline \multicolumn{5}{|l|}{ Melanoma } \\
\hline SKMEL3 & 1.3 & 0.5 & - & - \\
\hline A375 & 4.9 & 1.7 & - & - \\
\hline MEL-28 & $>20$ & $>7$ & - & - \\
\hline
\end{tabular}

activation of the CCND2 promoter in our $3 \mathrm{~T} 3$ reporter cells, demonstrated by an increase in LUC (Figure 8A). However, forskolin-induced upregulation of CCND2LUC was blocked by coincubation with kinetin riboside, indicating that kinetin riboside functions downstream of cAMP to inhibit CCND2 trans-activation. Similarly, treatment of $3 \mathrm{~T} 3$ with a cAMP phosphodiesterase inhibitor, RO-20-1724, also caused upregulation of CCND2LUC and was blocked by kinetin riboside, confirming that kinetin riboside inhibits cAMP pathway-induced CCND2 promoter trans-activation.

Protein phosphatase 2A (PP2A) is a negative regulator of the MAPK cascade (25) and other protein kinases. During screening, we determined that inhibition of PP2A using cantharidin caused marked upregulation of the CCND2 promoter in our LUC reporter model, suggesting that PP2A-related phosphoproteins play a regulatory role in CCND2 expression. However, as with cAMP-driven CCND2 promoter trans-activation, stimulation of the CCND2 promoter by cantharidin (Figure $8 \mathrm{~A})$ can be abolished by kinetin riboside, indicating that kinetin riboside blocks CCND2 promoter trans-activation at a level that is distal to both cAMP and the phosphoprotein targets of PP2A.

Kinetin riboside induces transcriptional repressors CREM1 $\alpha$ / $I C E R$, which can repress CCND1 and CCND2. Kinetin riboside suppresses cis activation of CCND1 (driven by relocation of the IGH enhancer) in U266 cells and also suppresses trans-activation of CCND2 in KMS11 and $\mathrm{H} 929$ cells (driven by overexpression of MAF and FGFR3); it furthermore suppresses both basal and MAFdriven trans-activation of the CCND2 promoter in $3 \mathrm{~T} 3$ cells and CCND2 trans-activation induced by cAMP- or PP2A-related phosphoprotein pathways (see Figures 2, 3 , and 8). Together, these findings suggest that cyclin D ultimately activate CCND2 - and from our results, we cannot exclude that cyclin D2 is more relevant to the survival of KMS11 tumor cells than cyclin D1.

Kinetin riboside requires adenosine kinase for full activity. Kinetin riboside is an adenosine derivative and may therefore enter nucleoside metabolic pathways. We next tested to determine whether kinetin riboside requires phosphorylation by adenosine kinase for activity (Figure 7). A-134974 is a specific inhibitor of adenosine kinase. At low concentrations, A-134974 has no effect on 3T3 cell viability and caused modest (1.4-fold) enhancement of expression of the reporter construct expressing firefly LUC from the human cyclin D2 promoter (CCND2-LUC) (Figure 7A). In contrast, A-134974 completely inhibited kinetin riboside-induced suppression of CCND2 promoter activity in $3 \mathrm{~T} 3$ reporter cells. Thus, kinetin riboside requires adenosine kinase and possibly phosphorylation for maximal activity. In $\mathrm{H} 929$ cells, A-134974 partially rescues cyclin D1 and D2 levels following kinetin riboside treatment and prevents kinetin-induced caspase cleavage (Figure 7B), confirming that adenosine kinase is required for full kinetin riboside activity.

Kinetin riboside blocks CAMP-and PP2A-induced CCND2 trans-activation. As kinetin riboside inhibits $C C N D 2$ promoter trans-activation by MAF and as CAMP signaling potently trans-activates CCND2 (24), we tested to determine whether kinetin riboside can inhibit cAMP-induced CCND2 promoter trans-activation. As expected, stimulation of the cAMP pathway with forskolin caused marked gene suppression by kinetin riboside may involve recruitment of a transcriptional repressor to CCND1 and CCND2 or inactivation of an essential transcription factor. As there is a 4- to 6-hour delay between kinetin riboside exposure and cyclin D1 or D2 suppression (see Figures 2 and 3), we surmised that changes in expression of an upstream gene may be required to effect cyclin $\mathrm{D}$ gene silencing.

We therefore performed gene-expression profiling on U266 and H929 cells 4 hours after treatment with kinetin riboside to identify effector molecules that mediate the kinetin riboside-induced blocks in CCND1 or CCND2 activation observed in both cell lines. At 4 hours, kinetin riboside induced a greater than 3 -fold change in the expression of only 36 genes in both H929 and U266 cells (Supplemental Table 2). Conspicuously, given its ability to block cAMP-mediated CCND2 trans-activation, kinetin riboside rapidly induced 3- to 5-fold upregulated expression of the cAMP-response element modulator gene (CREM). CREM encodes bZIP transcription factors that bind cAMP-response elements (CRE) in responsive gene promoters. Although CREM isoforms, of which there are more than 20, function either as activators or potent repressors of CRE-mediated transcription, kinetin riboside caused induction only of repressor isoforms (including the CREM type $1 \alpha$ repressor and inducible cAMP early repressor 1 [ICER1]) but failed to induce CRE activator isoforms (Supplemental Figure 4), suggesting a mechanism for its repression of select CRE-responsive genes, including CCND1 and CCND2. Upregulation of CREM repressor 
A

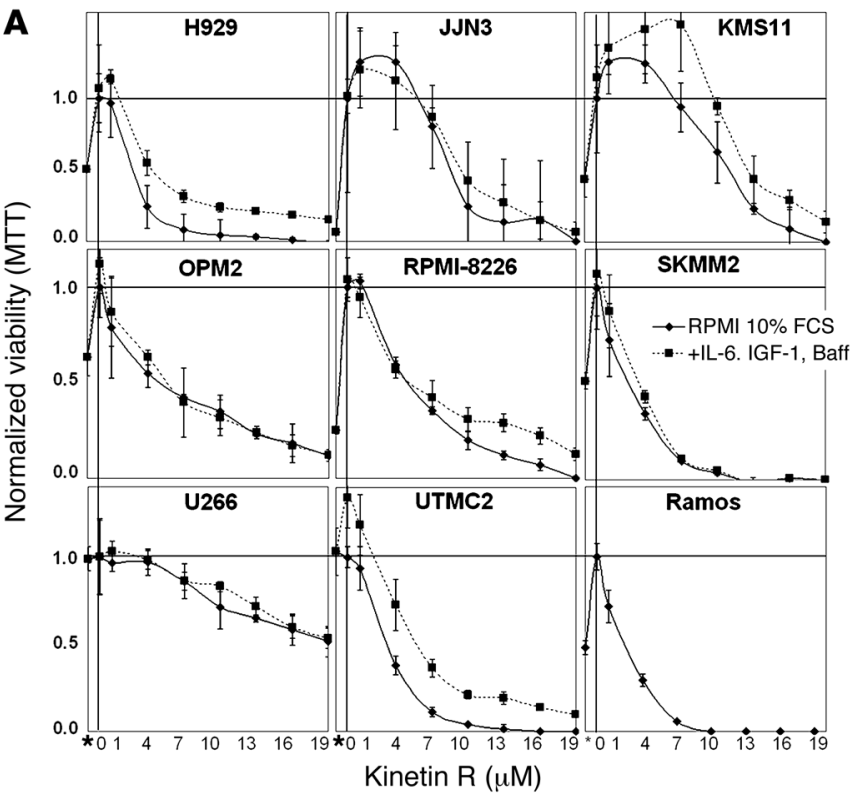

B

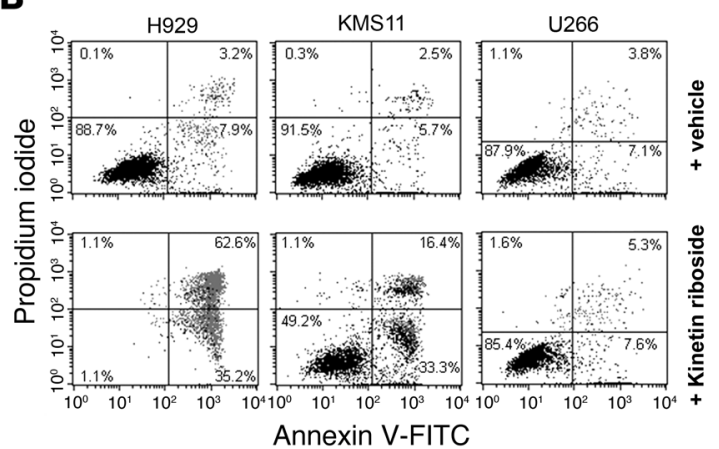

D

Examples of MM patient bone marrow samples treated with kinetin $\mathrm{R}$
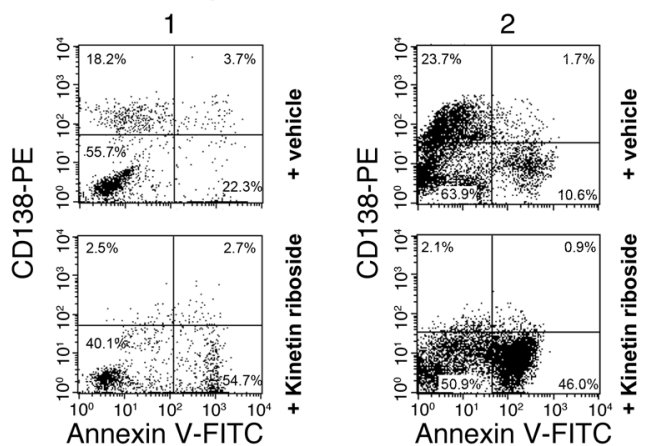

C No Drug

-Dexamethasone $\square$ Kinetin $\mathrm{R}$ $\square$ Dex + Kinetin R

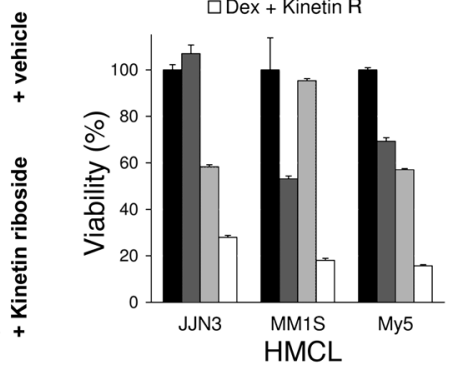

E

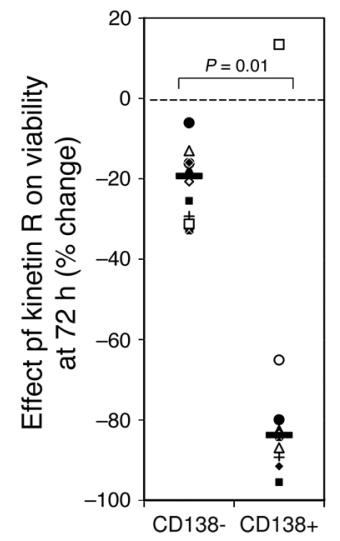

Figure 5

Kinetin riboside induces growth arrest and apoptosis in HMCL and primary myeloma cells and shows synergistic cytotoxicity with corticosteroids. (A) HMCL viability following kinetin riboside $(1-20 \mu \mathrm{M})$ in the presence or absence of MM growth cytokines IL-6 (10 ng/ $\mathrm{ml})$, IGF-1 (100 ng/ml), and BAFF $(25 \mathrm{ng} / \mathrm{ml})$ by MTT assay at 72 hours. The Burkitt lymphoma line RAMOS is also shown. *MTT on day 0 without drug. (B) Induction of apoptosis in HMCL by vehicle or kinetin riboside at 96 hours, assessed by annexin $\mathrm{V}$ and propidium iodide uptake. (C) Synergistic cytotoxicity induced by kinetin riboside $(5 \mu \mathrm{M})$ and dexamethasone (10 nM) in JJN3, MM1S, and MY5 HMCL, assessed by MTT assay at 48 hours; graph shows the mean of triplicates \pm SEM. (D) Two examples of flow cytometric analyses of MM patient bone marrow samples treated with vehicle or kinetin riboside $(10 \mu \mathrm{M})$, showing preferential loss of viable primary CD138+ tumor cells (left upper quadrants) versus CD138- hematopoietic cells (left lower quadrants) with kinetin riboside at 72 hours. Following kinetin riboside treatment, CD138+ plasma cells became annexin $\mathrm{V}$ positive and CD138 negative, consistent with apoptosis. (E) Effects of kinetin riboside on $\mathrm{CD}_{138^{+}}$and CD138- compartments in $10 \mathrm{MM}$ patient bone marrow samples at 72 hours. CD138+ and CD138- viability responses are compared by Mann-Whitney test. CD138+ (primary tumor) cells are preferentially killed. isoforms by kinetin riboside was next verified by quantitative PCR (Figure 8B) using primers that target the isoforms (ICER1 and CREM $1 \alpha$ ) that gene-expression analysis had revealed were expressed at increased levels; 3 - to 6-fold induction of these CREM transcripts by PCR in H929 and U266 MM cells was detected, consistent with the results of gene-expression profiling.

Expression of cyclin D1 in MM has previously been shown to be critically dependent upon a CRE site in the CCND1 promoter (26);
CCND2 is similarly responsive to cAMP via a CRE site in its own promoter and responds to CREM repressor control (24). In KMS11 myeloma cells, plasmid-mediated expression of the induced CREM repressor cDNA caused suppression of CCND2 promoter activity, measured by LUC reporter (Figure 8C), supporting a role for kinetin riboside-induced CREM repressor expression in the modulation of CCND2 promoter activity by kinetin riboside. Notably, an evolutionarily conserved CRE/activating transcription factor/acti- 
A

KMS11

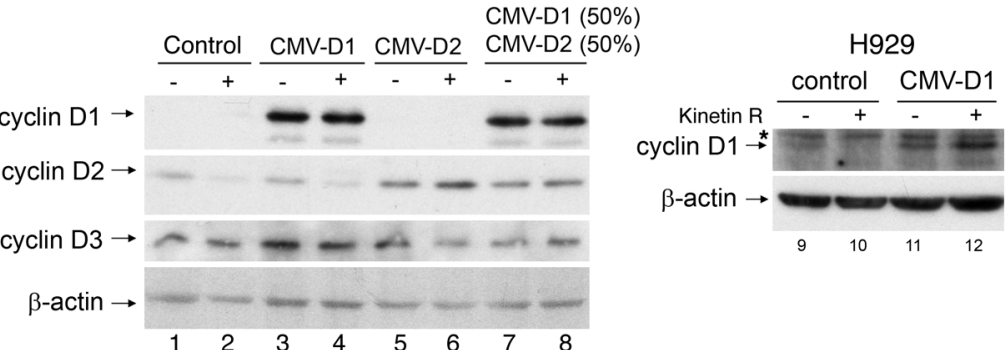

B

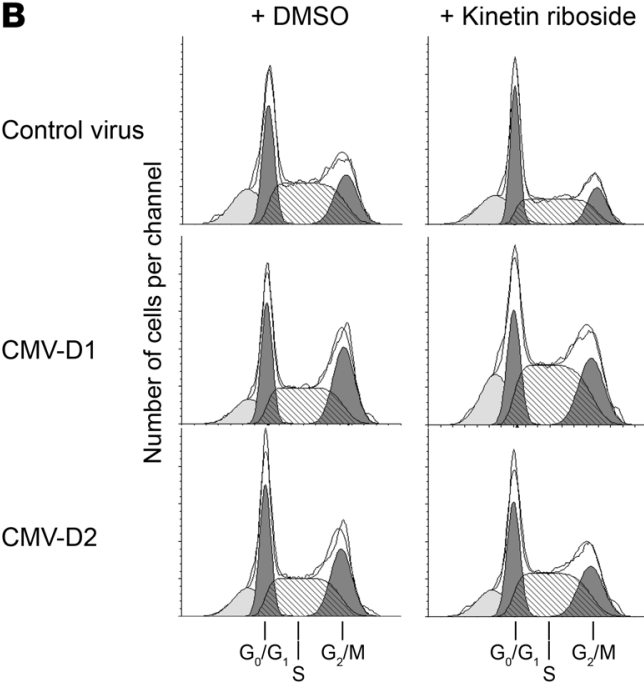

Cell cycle stage (FL2-A channel)
D

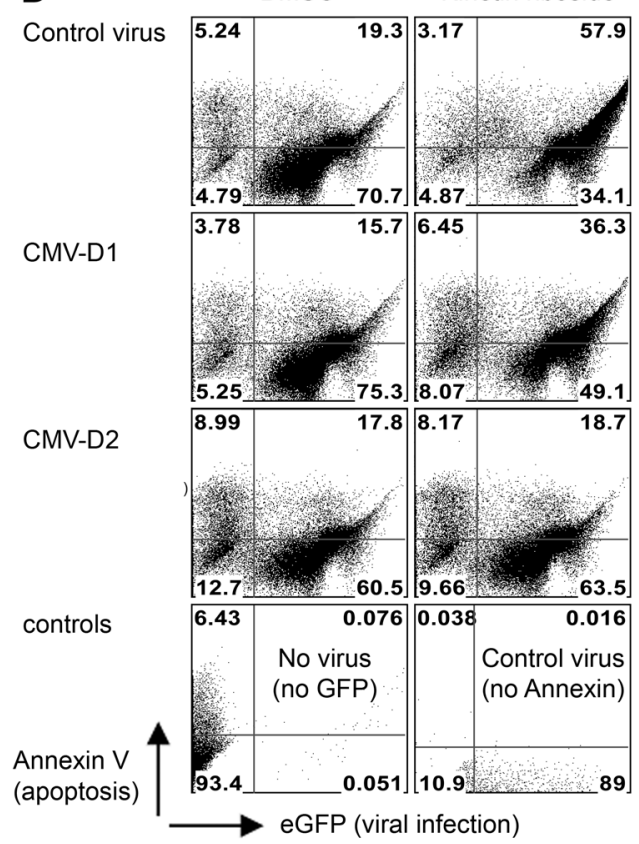

E

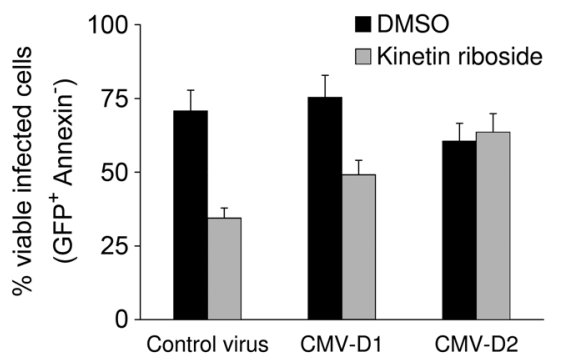

Figure 6

Cyclin D1 or D2 expressed from a heterologous CMV promoter is not suppressed by kinetin riboside and rescues KMS11 cells. (A) Adenoviruses inducing expression of eGFP (control), cyclin D1 (CMV-D1), or cyclin D2 (CMV-D2) from heterologous CMV promoters were used to infect KMS11 or H929 cells; 48 hours later, cells were aliquotted and treated with DMSO vehicle (-) or kinetin riboside (15 $\mu \mathrm{M})(+)$. Lysates were prepared at 12 hours; immunoblots show cyclin D protein levels following kinetin riboside treatment. Endogenously expressed cyclin D2 (lanes 1 and 3) and D1 (lane 9) is suppressed by kinetin riboside (lanes 2, 4, and 10), while CMV promoter-expressed cyclin D2 or D1 is not suppressed. (B) Cell-cycle studies on aliquots of cells from A were performed 20 hours after kinetin riboside treatment. The results are from 1 of 2 similar experiments. Expression of cyclin D1 or D2 from the heterologous CMV promoters rescues KMS11 cells from kinetin riboside-induced $\mathrm{G}_{0} \mathrm{G}_{1}$ cell-cycle arrest. (C) The proportion of KMS11 cells undergoing cell division following kinetin riboside treatment is summarized for cells expressing cyclin D1 or D2 from endogenous or CMV promoters, with an estimated 10\% error. (D) Flow cytometry on adenovirus-infected KMS11 cells depicted in $\mathbf{A} 72$ hours after treatment with vehicle or kinetin riboside (15 $\mu \mathrm{M})$ showing infection status (eGFP expression) and viability (annexin V binding), comparing cells expressing cyclin D1 or D2 from endogenous or heterologous promoters. (E) Summary of kinetin riboside-induced apoptotic responses in KMS11 cells expressing cyclin D1 or D2 from endogenous or heterologous (CMV) promoters. Graph shows the measured percentage of viable (annexin $\mathrm{V}^{-}$) virus-infected (GFP+) cells $\pm 10 \%$ error (estimated). 
A
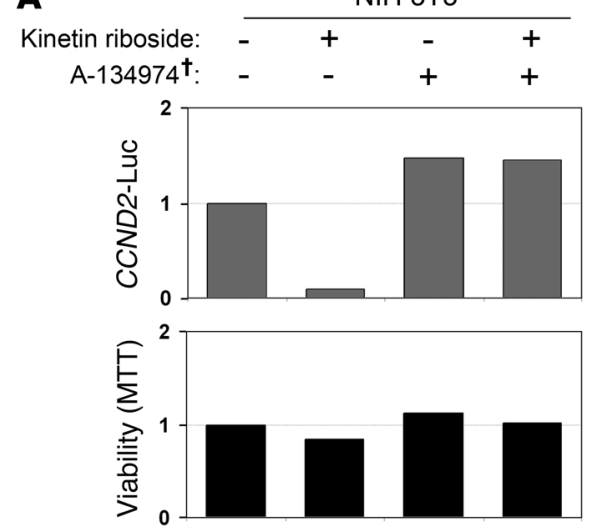

B

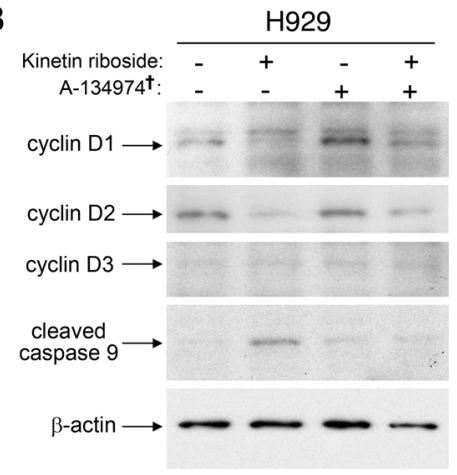

vator protein 1 (CRE/ATF/AP-1) multitranscription factor-binding site is present in both CCND2 and CCND1 promoters immediately upstream $(<50 \mathrm{bp})$ of their transcription origin $\left(5^{\prime} \mathrm{UTR}\right)$ but is absent in CCND3, providing a rationale for the specificity in cyclin $\mathrm{D}$ response to kinetin riboside.

In addition to inducing CREM repressors, kinetin riboside also rapidly induced the transcription repressor BTB and CNC homology 1 basic leucine zipper transcription factor $2(\mathrm{BACH} 2)$ in both $\mathrm{H} 929$ and $\mathrm{U} 266$ tumor lines. Induction of BACH2, which was first detected by gene-expression analysis (Supplemental Table 2), was also verified by quantitative PCR (Figure $8 \mathrm{~B}$ ). BACH2 is a known repressor of MAF and AP-1 transcription factor sites $(27,28)$ and of the $3^{\prime}$ IGH enhancer and therefore may also contribute to kinetin riboside-induced cyclin D gene suppression (discussed below).

Kinetin riboside inhibits growth of human myeloma xenograft tumors in nude mice. The antitumor activity of kinetin riboside in vivo was evaluated against MY5 and 8226 human myeloma tumors grown as xenografts in nude mice. These myeloma tumor lines both dysregulated $M A F$ via $\mathrm{t}(14 ; 16)$ translocations to trans-activate CCND2 (2). Drug or vehicle were initially administered once daily with dosing up to $100 \mathrm{mg} / \mathrm{kg} / \mathrm{d}$; however, no effect on 8226 (not shown) or MY5 (Figure 9A) tumor growth was observed. Nevertheless, as the limiting toxicity for single-dose escalation was transient mild sedation with short duration (half-life of approximately 15 minutes), an intensified multidosing regimen was subsequently instituted to counter rapid drug redistribution or clearance. Consistent with a short drug half-life, rapidly repeated doses of kinetin riboside (up to 5 per day at $85 \mathrm{mg} / \mathrm{kg} /$ dose) were well tolerated. Moreover, with progressive dose-density intensification in MY5-xenografted mice, tumor stasis was observed at $4 \times$ daily dosing and regression of tumors was observed at $5 \times$ daily dosing, despite substantial pre-

\section{Figure 7}

Kinetin riboside requires adenosine kinase for activity. (A) Kinetin riboside-induced suppression of CCND2 promoter activation in 3T3 cells is abolished by the adenosine kinase inhibitor A-134974 (2 $\mu \mathrm{M})$. 3T3 cells containing the CCND2 promoter-LUC reporter construct were preincubated with $\mathrm{A}-134974(2 \mu \mathrm{M})$ for 1 hour and were then treated with kinetin riboside $(10 \mu \mathrm{M})$ for 16 hours; CCND2 trans-activation and viability were determined by LUC and MTT assays, respectively. tSpecific adenosine kinase inhibitor. (B) Immunoblot of H929 MM cells showing that kinetin riboside-induced $(10 \mu \mathrm{M})$ cyclin $\mathrm{D}$ suppression and caspase- 9 cleavage are diminished by A-134974 $(2 \mu \mathrm{M})$.

ceding tumor growth (Figure 9A). Xenograft studies were repeated with 8226 tumors using kinetin riboside $(85 \mathrm{mg} / \mathrm{kg} 4 \times$ daily up front), started when tumors had reached a mean volume of 135 $\mathrm{mm}^{3}$ (Figure 9B). Statistically significant tumor growth inhibition was demonstrated compared with that in vehicle-treated mice $(P<0.01)$. No effects on neutrophil counts (at 14 days treatment) or on body weight were observed.

\section{Discussion}

Cytokinins are plant hormones that activate cytokinesis in quiescent plant cells by induction of cyclin D3 gene trans-activation, causing expression of cyclin D3 (21). We find here that the synthetic cytokinin, kinetin riboside, has the reciprocal activity in mammalian cells and induces suppression of cyclin D1 and D2 but does not significantly affect cyclin D3.

Kinetin riboside acts in mammalian tumor cells to inhibit transcription from the CCND2 promoter and notably blocks transactivation induced by cAMP or by regulatory phosphoproteins. In $3 \mathrm{~T} 3$ cells, kinetin riboside suppresses CCND2-LUC signal in a manner that is dependent upon the presence of the CCND2 promoter but that does not require the cyclin D2 mRNA coding sequence, indicating regulation of CCND2 promoter activity, rather than posttranscriptional function. Similarly, kinetin riboside causes suppression of cyclin D1 and D2 when these are expressed from their endogenous promoters in myeloma cells but does not suppress cyclin D1 or D2 when these are expressed from a heterologous CMV promoter, indicating that kinetin riboside modulates cyclin D levels via the CCND1 or CCND2 promoters alone and does not cause cyclin $\mathrm{D}$ suppression from nonspecific cytotoxicity or from posttranslational degradation. Furthermore, kinetin riboside effectively suppresses CCND2 when this gene is trans-activated by overexpression of MAF (which directly binds the CCND2 promoter), suggesting that CCND2 suppression by kinetin riboside involves recruitment of a transcriptional repressor to the CCND2 promoter or suppression of an essential cofactor required for CCND2 transcription.

From gene-expression profiling studies, we show that kinetin riboside induces repressor isoforms of the CRE modulator gene. We (above) and others $(24,29)$ have found that cAMP signaling has a potent effect on CCND2 transcription, which is mediated by a conserved CRE site located immediately $5^{\prime}$ to the transcriptional origin (29). CREM repressors regulate CCND2 by binding this CRE (24). Repressor isoforms of CREM share the DNA binding and dimerization domains of CREM activators but lack kinase and trans-activation domains and therefore act as potent dominant negative transcription inhibitors (30-32). CREM repressor activity is regulated by gene expression rather than by posttranslational modification $(32,33)$. A functional CRE is also present in 


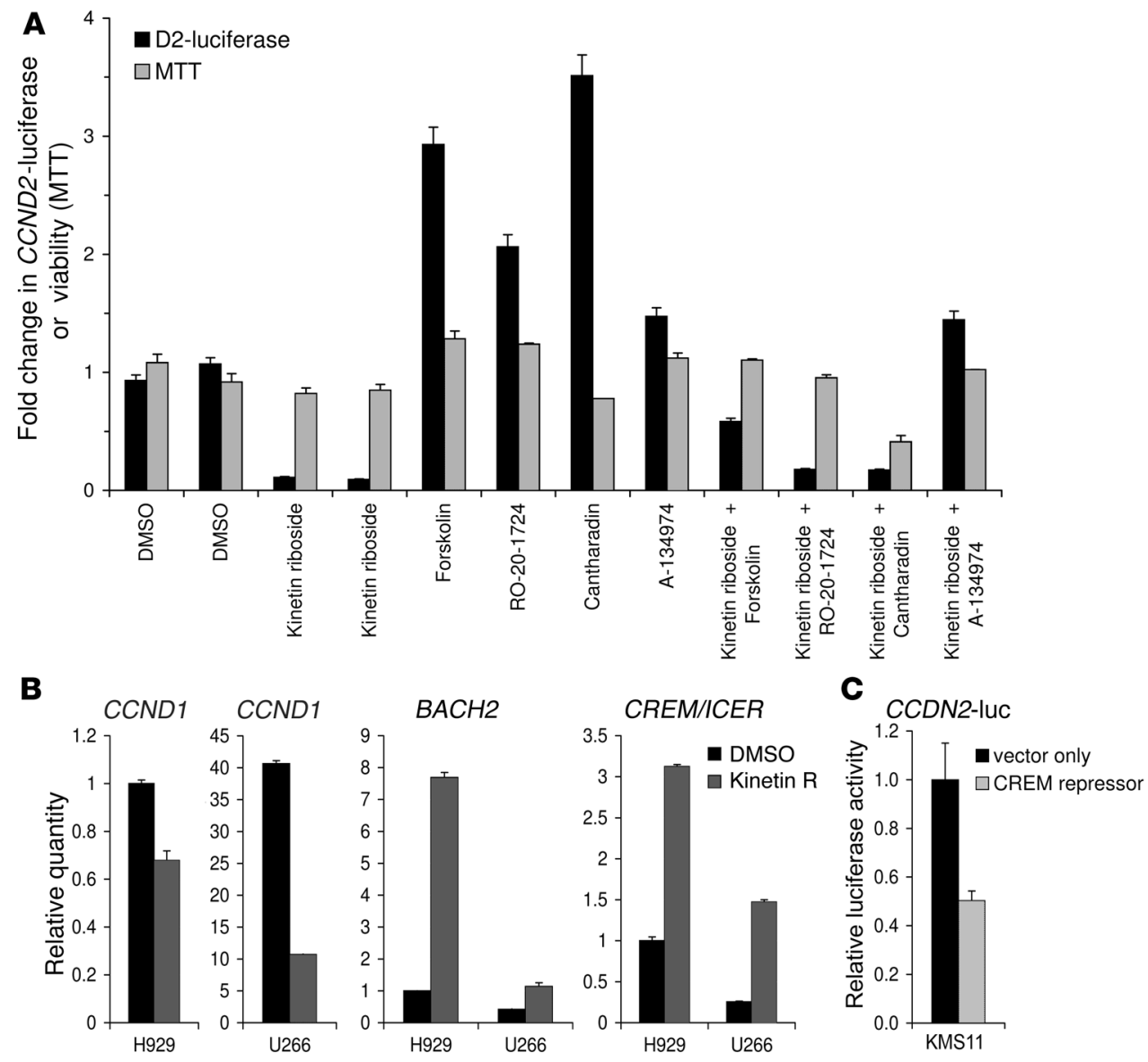

Figure 8

Kinetin riboside induces transcriptional repressors $C R E M$ and $B A C H 2$ and blocks CAMP- and PP2A-induced CCND2 promoter activation. (A) 3T3 CCND2-LUC reporter cells were incubated with kinetin riboside or vehicle plus or minus forskolin, RO-20-1724, cantharidin, or A-134974, as specified $(5 \mu \mathrm{M})$ for 16 hours; CCND2 trans-activation and viability were then assessed. Forskolin activates adenylate cyclase; RO-20-1724 inhibits CAMP phosphodiesterase; both cause increased cAMP signaling, which is seen to activate the CCND2 promoter. Cantharidin inhibits PP2A and also stimulates CCND2 promoter transcription. As shown, kinetin riboside blocks CCND2 promoter trans-activation by cAMP or PP2Arelated phosphoproteins, initiated by forskolin, RO-20-1724, or cantharidin. Results represent 1 of 2 experiments and are shown as the mean of duplicate samples \pm SEM. (B) H929 and U266 myeloma cells were treated with kinetin riboside $(10 \mu \mathrm{M})$ for 4 hours and then processed for quantitative RT-PCR. Relative expression of genes determined by RT-PCR was normalized to H929 cells treated with vehicle alone and is plotted as the mean of replicates; error bars show the range. Consistent with gene-expression profiling analysis, quantitative RTPCR confirms that kinetin riboside rapidly induces $C R E M$ and $B A C H 2$ transcriptional repressors in both $\mathrm{H} 929$ and U266; parallel early suppression of CCND1 is also shown. (C) A pCMV-SPORT6 vector expressing the induced CREM repressor cDNA or a control pCMV-SPORT6 vector was cotransfected with the CCND2-LUC reporter into KMS11 myeloma cells, and CCND2 promoter activity was determined by LUC assay at 30 hours. Expression of the CREM repressor caused suppression of CCND2 promoter activity.

CCND1 (34-36) at a location identical to that in CCND2; however, an analogous CRE is not present in CCND3. Together, these observations demonstrate a mechanism by which kinetin riboside can specifically suppress cyclin D2 and D1, but not D3, blocking gene transcription induced by diverse direct or indirect cis- or trans-activating signals (Supplemental Figure 5).

A common misconception is that cyclin D gene expression is suppressed by default in cells subjected to cytotoxic stimuli. In fact, we find that in cells treated with conventional cytotoxics, CCND2 gene transcription continues at levels consistent with the cell-cycle stage at which cells arrest ( $\mathrm{R}$. Tiedemann, unpublished observations). Thus, cells pharmacologically induced to arrest in S-phase show low CCND2 transcriptional activity (consistent with normal S-phase), while cells treated with cytotoxics active in
$\mathrm{G}_{2} / \mathrm{M}$ phase show persistently upregulated CCND2 transcription (consistent with the $\mathrm{G}_{2} / \mathrm{M}$ phase of actively dividing cells). This suggests that, at least for many conventional cytotoxics, cyclin D gene transcriptional activity is irrelevant to the mechanics of cell death. Importantly, kinetin riboside differs from conventional cytotoxics such as camptothecin that can be indirectly linked with low cyclin D expression as a result of first inducing S-phase arrest. Instead, kinetin riboside induces rapid suppression of cyclin D1 and D2 (<6 hours) and cells subsequently arrest in $\mathrm{G}_{0} / \mathrm{G}_{1}$ when cyclin $\mathrm{D}$ is required for cell-cycle progression. Reexpression of cyclin D1 or D2 from a kinetin riboside-unresponsive heterologous promoter rescues cells from kinetin ribosideinduced proliferative arrest and, at least for KMS11 cells, from apoptosis. The same is unlikely to be true for many unrelated 

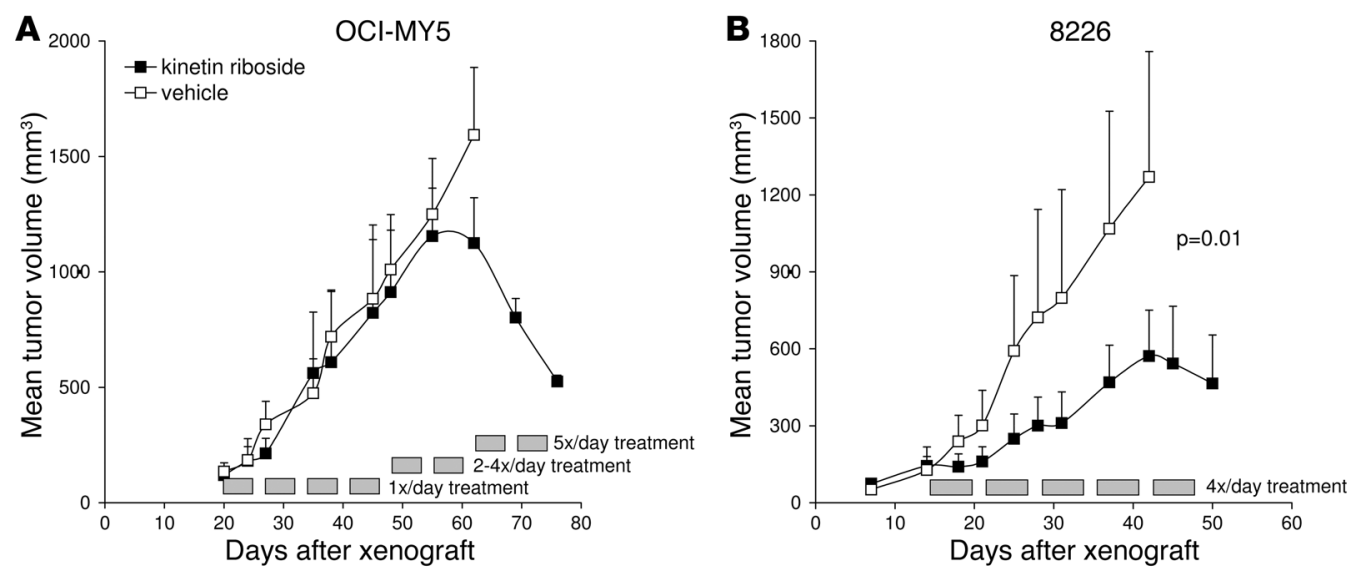

Figure 9

Kinetin riboside induces tumor growth arrest in MY5 and 8226 myeloma tumor xenografts. (A) Matched pairs of nude mice bearing MY5 tumors were treated i.p. with vehicle control (open squares) or with kinetin riboside (filled squares) at a dose density escalating from $100 \mathrm{mg} / \mathrm{kg}$ once daily to $85 \mathrm{mg} / \mathrm{kg} 5 \times /$ daily, administered 5 days per week, commencing after mean tumor volume reached $135 \mathrm{~mm}^{3}$. Mean tumor volumes $\pm \mathrm{SEM}$ are shown from the time of xenograft initiation ( $n=4 /$ group). (B) Nude mice bearing 8226 tumors were treated with vehicle (open squares) or with kinetin riboside (filled squares) ( $n=4 /$ group) commencing at $85 \mathrm{mg} / \mathrm{kg} 4$ times daily (i.p. and s.c.) when the mean tumor volume exceeded $135 \mathrm{~mm}^{3}$. $P$ value was calculated by paired $t$ test.

conventional cytotoxic agents that influence cyclin D protein levels only after first inducing cytotoxicity.

Treatment of myeloma cells with kinetin riboside not only induced suppression of cyclin D1 and D2 and $G_{0} / G_{1}$ phase arrest but in many HMCL and primary myeloma samples was associated with induction of apoptosis. The mechanism of apoptosis induction remains unclear but may in part be due to a need for continued cell division to maintain malignant cell viability. RNA interference experiments suggest that cyclin D1 \pm D2 silencing and $\mathrm{G}_{0} \mathrm{G}_{1}$ arrest can eventually be lethal to myeloma cells. Importantly, cyclin D3 levels do not appear to be significantly affected by kinetin riboside treatment. Despite this, kinetin riboside induces $\mathrm{G}_{1}$ arrest and blocks entry of myeloma cells into the cell cycle. While cyclin D3 is important in early B cell development, it is expressed at only low levels in plasma cells and $\mathrm{B}$ cells beyond the pre-B stage (37) and is dysregulated in only a minority of MM patients (3\%) with 6p21 translocations (2). Basal expression of cyclin D3 in MM cells may simply be insufficient to sustain cell division after drugor shRNA-induced suppression of oncogenetically dysregulated cyclin D1 or D2.

Kinetin riboside may exert cellular effects that promote apoptosis in addition to regulating cell-cycle entry. In plants, cytokinins control many aspects of plant development $(19,20)$ and also induce apoptosis of plant tumor cells (38) as well as regulating cell division. Others have recently investigated the differentiating activities of plant cytokinins against human cancers (38-40) and have noted growth inhibition that may in part be accounted for by suppression of cyclin Ds. In addition, several cytokinins, including kinetin riboside, have been shown to cause alterations in mitochondrial membrane potential as a prelude to apoptosis (40).

In addition to inducing transcriptional repressors of CRE, kinetin riboside caused prompt upregulation of the repressor $\mathrm{BACH} 2$ in both $\mathrm{H} 929$ and $\mathrm{U} 266$ cells. BACH2 is a B cell-specific partner for small MAF family proteins and functions as a generic repressor of MAF response element (MARE) sites (27). Induction of BACH2 by kinetin riboside may partly explain the blockade of MAF-induced
CCND2 trans-activation seen with this compound. In diffuse large cell lymphoma, where CCND2 overexpression by gene-expression profiling predicts poor prognosis, expression of $B A C H 2$ predicts good prognosis (41). Significantly, $\mathrm{BACH} 2$ negatively regulates the immunoglobulin $3^{\prime}$ enhancer $(27,28,42,43)$ and may therefore also inhibit expression of oncogenes dysregulated by translocations to this enhancer, including (in MM) CCND1, FGFR3, or MAF.

Notably, kinetin riboside can be isolated from coconut milk and is therefore present in the human food chain, albeit at low concentrations $(0.2 \mathrm{nM})(44)$. The free base, kinetin, has been isolated in human urine at near similar concentrations (45). Moreover, kinetin is widely used in topical cosmetics (46-48) due to reports that it prevents morphological and nuclear changes associated with "cellular aging." Despite these encouraging coincidences, it remains to be determined whether this exciting cytotoxic agent or potential derivatives have a therapeutic window that will permit targeted treatment of patients with malignancies exhibiting dysregulation of cyclin D1 or D2 in the clinic.

\section{Methods}

Cell culture and cytokines. HMCL were grown in RPMI. Primary MM and bone marrow cells were maintained in Iscove's modified Dulbecco's medium. NIH3T3 and 293T were cultured in DMEM. Cultures were supplemented with $10 \%$ fetal bovine serum and $1 \mathrm{mM}$ glutamate; primary cells were also supplemented with $10 \mathrm{ng} / \mathrm{ml}$ IL-6. Where indicated, HMCL were supplemented with IL-6, IGF-1, and BAFF (R\&D Systems).

Primary $\mathrm{CD} 138^{+}$myeloma cell purification. Bone marrow from MM patients was obtained with Mayo Clinic institutional review board approval and in accordance with the Declaration of Helsinki. Bone marrow samples were treated by ACK lysis to remove red cells, and where required, primary $\mathrm{MM}$ cells were further purified by $\mathrm{CD} 138^{+}$immunomagnetic sorting as described (2).

Lentiviral vectors and shRNA-induced silencing of cyclin D1 and cyclin D2. TRC consortium lentiviral vectors directing the expression of CCND1-, CCND2or NT-shRNA were obtained as sequence-verified bacterial stocks (SigmaAldrich) and prepared by transfection of plasmid DNA in 293T cells as 
described $(49,50)$. Virus was harvested at 36 hours. Eight lentiviruses targeting CCND1 or CCND2 were screened to identify shRNA that optimally suppressed cyclin D1 or D2; immunoblotting of H929 lysates 48 hours after infection was used to gauge suppression of cyclin D relative to $\alpha$-tubulin. Lentivirus clones that optimally suppressed cyclin D1 or D2 are listed in Supplemental Table 1 and were used for subsequent experiments. In functional studies, H929 cells were infected with equal quantities of lentivirus in the combinations specified. Cyclin D silencing was verified by immunoblot at 60 hours; the same cultures were examined for cell-cycle transit and for apoptosis as specified.

Genetic constructs for stimulating and assaying CCND2 trans-activation. MAF cDNA was subcloned into an internal ribosome entry site-enhanced GFP murine stem cell virus-based retroviral vector and introduced into NIH3T3 cells by infection. Stable clones expressing GFP and MAF were selected by flow cytometry and immunoblotting. The human CCND2 promoter (-894 to -4) (51) containing a MARE was cloned from HeLa DNA and inserted into pGL2basic LUC vector (Promega) immediately upstream of the LUC gene. This construct was cotransfected with pcDNA3-encoding neomycin resistance into both 3T3 wild-type cells and 3T3 cells overexpressing MAF-internal ribosome entry site-GFP. Cells stably expressing MAF, GFP, and LUC were selected.

High throughput screen for inhibitors of CCND2 trans-activation. 3T3 CCND2LUC reporter cells were dispensed into 96-well plates (13,000/well) with a Biomek FX liquid handler (Beckman) and, following adherence, were treated with compounds from LOPAC (Sigma-Aldrich), Prestwick (Prestwick Chemical), or Spectrum (Microsource) libraries at final concentrations of approximately $5 \mu \mathrm{M}$ with $<0.1 \% \mathrm{DMSO}$ at $37^{\circ} \mathrm{C}$ for 16 hours. After incubation, $C C N D 2$ activation was assessed by LUC assay and viability was assessed by MTS assay.

Luc assay. Luc activity was assessed according to the substrate manufacturer's instructions (Promega). In brief, culture media was removed with an EMBLA plate washer and Glo Lysis Buffer was added by robot. After 10 minutes, an equal volume of Bright-Glo Luciferase substrate was added, and luminescence was detected with a Luminoskan plate reader (Thermo Labsystem) using 5-second integration.

MTS and MTT viability assays. For screening, cell viability was assessed by MTS assay (Promega). MTS reagent $\left(20 \mu \mathrm{l} /\right.$ well) was added at $37^{\circ} \mathrm{C}$ and incubated for 4 hours, and absorbance at $490 \mathrm{~nm}$ was determined. For follow-up studies, viability of HMCL was determined by MTT assay (SigmaAldrich) following the manufacturer's instructions.

Functional studies of kinetin riboside effects on the CCND2 promoter. CCND2 suppression by kinetin riboside was examined by cotreatment of 3T3 reporter cells with drugs (all purchased from Sigma-Aldrich) that influence CCND2 trans-activation or inhibit nucleoside phosphorylation. Reporter cells were incubated with kinetin riboside or vehicle, with or without forskolin, 4-(3-butoxy-4-methoxybenzyl)imidazolidin-2-1 (RO-20-1724), cantharidin, or A-134974, as specified, at $5 \mu \mathrm{M}$ for 16 hours at $37^{\circ} \mathrm{C}$; CCND2 activity and viability were assessed as described above. Forskolin is a diterpenoid that activates adenylate cyclase and increases cAMP. RO-20-1724 is an inhibitor of cAMP phosphodiesterase that also causes accumulation of cAMP. Cantharidin is an inhibitor of PP2A. A-134974 is a selective inhibitor of adenosine kinase.

Immunoblotting. Cell lysate preparation, gel electrophoresis, and immunoblotting were performed using standard techniques (52). PVDF membranes were probed with antibodies against cyclin D1 (DCS-6; Biosource), cyclin D2 (2924; Cell Signaling Technology), cyclin D3 (DCS-22; BioSource), MAF (M-153; Santa Cruz Biotechnology Inc.), cleaved caspase-9 (Asp315, 9505; Cell Signaling Technology), $\beta$-actin (4967; Cell Signaling Technology), and $\alpha$-tubulin (B-7; Santa Cruz Biotechnology Inc.). Proteins were visualized by chemiluminescence (Pierce).
Annexin V flow cytometry. Cells were washed in cold PBS. For 2-color analyses, cells were resuspended in buffer containing annexin V-Alexa Fluor 488 (A13201; Invitrogen) and propidium iodide (BD Biosciences) or anti-CD138-PE (552026; BD Biosciences), as specified. For 3-color analyses, cells were resuspended in buffer containing annexin V-Alexa Fluor 488 (A13201; Invitrogen), anti-CD45-PECy7 (H130; BD Biosciences), and antiCD38-PE (555460; BD Biosciences). After 15 minutes, cells were diluted and analyzed immediately by FACScan (BD Biosciences).

Cell-cycle studies. Cells were resuspended in ice-cold PBS, and ethanol was added dropwise to $75 \%$. Samples were incubated at $-20^{\circ} \mathrm{C}$ for 1 hour, washed with PBS, and resuspended in $100 \mu \mathrm{g} / \mathrm{ml}$ RNAse A. Propidium iodide $(20 \mu \mathrm{g})$ was added immediately prior to flow cytometry. Cellular aggregates were excluded using CellQuest Doublet Discriminator Module, and cell-cycle profile was assessed with ModFit LT for Mac 3.1 (Verity Software).

Generation of helper-dependent adenoviral vectors directing expression of CCND1 and CCND2 from a heterologous CMV promoter. Helper-dependent adenoviral vectors expressing cyclin D1 or cyclin D2 from a heterologous CMV promoter and enhanced GFP from a separate promoter were constructed from the previously described adenoviral precursor plasmid, pSC27B (53), which encodes GFP. We created a PSC11HCPA shuttle plasmid by introducing a human CMV multicloning site-BGH poly-A cassette between I-Sce I and I-Ceu I restriction sites of PSC11 (54), allowing a gene-of-interest expression cassette from PSC11HCPA to be transferred to the right inverted terminal repeat region of pSC27B. Cyclin D1 and D2 were amplified by PCR (CCND1F: 5'-TCAGATCTATGGAACACCAGCTCCTGTG-3'; CCND1R: 5'-ATAGATCTTCAGATGTCCACGTCCCGCA-3'; CCND2F: 5'-AGATCTATGGAGCTGCTGTGCCACGA-3'; CCND2R: 5'-AGATCTTCACAGGTCGATATCCCGCAC-3'), sequence verified, and cloned separately into pSC11HCPA under the control of a human CMV promoter. The expression cassettes were excised with I-Ceu I and I-Sce I and transferred to pSC27B to create pSC27B-CCND1 and pSC27B-CCND2, respectively. Helper-dependent adenoviral particles were prepared by repeated cycles of amplification in 293Cre 4 cells (kindly provided by Frank Graham, McMaster University, Hamilton, Ontario, Canada) with the adeno helper virus AdRP2050 (Robin Parks, Ottawa Health Research Institute, Ottawa, Ontario, Canada), which contains a modified capsid with sequence RGD in the H1 loop of the fiber. Virus particles were purified using a 1-hour step gradient and overnight continuous $\mathrm{CsCl}$ gradient and then quantified (54). Myeloma cells were infected with 200 particles per cell in 60 -mm Petri plates with $2.5 \times 10^{6}$ cells in $5 \mathrm{ml}$ of medium. The percentage of infected cells was assessed by FACS analysis of the coexpressed GFP marker.

Gene-expression profiling. H929 and U266 HMCL were treated with kinetin riboside $(10 \mu \mathrm{M})$, an unrelated cytotoxic pristimerin $\left(0.5 \mu \mathrm{M} ; 2 \times \mathrm{IC}_{50}\right)$, or DMSO for 4 hours. RNA was isolated using Trizol, column purified (QIAGEN), and hybridized to Humn Genome U133 Plus 2.0 microarrays (Affymetrix) as previously described (2). Gene-expression intensity values were log transformed, normalized by chip and by cell line to DMSO-control-treated samples, and analyzed using GeneSpring 7 (Agilent Technologies). Genes were filtered by MAS5 detection calls to exclude nonexpressed probe sets with raw hybridization intensities of less than 20 and absent calls across all samples tested (treated and untreated).

CREM isoform transcript expression. Affymetrix probe sets targeting CREM gene expression were correlated with activator and repressor variant transcripts using Ensembl (55), Human ContigView v39 (www.ensembl.org/Homo_sapiens), and NCBI plus Sanger Vega (56) mRNA transcript databases.

Quantitative real-time PCR. Gene-expression analyses were verified by real-time PCR using TaqMan gene-expression assays (Applied Biosystems) on an ABI Prism 7700 (Applied Biosystems). Assays details are as follows: BACH2 (Hs00222364_m1); CREM - including NM_182717.1 and 
NM_183013.1 repressor isoforms found to be increased by gene-expression analyses (Hs01582006_m1); CCND1 (Hs00277039_m1); and $\beta$-actin (human $\beta$-actin VIC-MGB assay kit). Expression levels were compared by $\Delta \Delta C T$, where $\Delta \Delta C T=\Delta C T$ - (calibrator $\Delta C T)$. In this equation, $\Delta C T=$ average $C T$ - average $C T_{\beta \text {-actin. }}$. Relative quantities were calculated as $2^{-\Delta \Delta C T}$.

CCND2-LUCreporter activity in KMS11 cells. The cyclin D2 promoter reporter vector pGL2-CCND2-LUC ( $2 \mu \mathrm{g} /$ well) and either a PCMV-SPORT6 control vector $(8 \mu \mathrm{g} /$ well $)$ or an expression vector expressing inducible CREM repressor cDNA (8 $\mu \mathrm{g} /$ well, pCMV-SPORT6; Open Biosystems) were combined with Lipofectamine 2000 in $500 \mu \mathrm{l}$ serum-free media, incubated for 20 minutes, and cotransfected into adherent KMS11 cells cultured in antibiotic-free medium in a 6-well plate. The CREM repressor vector was used in excess of reporter plasmid to ensure coexpression of the repressor in cells transfected with reporter plasmid. CCND2-LUC activity was assayed at 30 hours as described above.

In vivo xenograft studies. Female nude $\mathrm{X}$-linked, immune-deficient mice purchased from NCI-Frederick were used for in vivo studies. Animals were approved for use in these experiments by the Mayo Clinic Institutional Animal Care and Use Committee. Eight-week-old mice were inoculated with MY5 or $8226\left(2 \times 10^{7}\right)$ MM cells s.c. in the right flank. Kinetin riboside (predissolved in DMSO at $500 \mathrm{mg} / \mathrm{ml}$ and stored at $-20^{\circ} \mathrm{C}$ ) was formulated daily by dropwise dissolution to $3.7 \mathrm{mg} / \mathrm{ml}$ in prewarmed $\left(37^{\circ} \mathrm{C}\right) 0.9 \%$ saline, $1 \% \beta 2$-hydroxypropylcyclodextrin (Sigma-Aldrich) solution and was administered between 1 and 5 times daily at $85 \mathrm{mg} / \mathrm{kg} / \mathrm{dose}$. The first daily dose was given i.p., and remaining doses were given s.c. with increasing dosing intervals $(30,60,120$, and 180 minutes). Tumor volumes were measured using vernier calipers and calculated with the formula $\mathrm{L} \times \mathrm{W} \times \mathrm{H} \times 2 / 3$. $P$ values were calculated by paired $t$ test (JMP 6.0; SAS).

\section{Acknowledgments}

A.D. Schimmer is a Canadian Institutes for Health Research clinician scientist. R.E. Tiedemann was funded in part by the Haematology Society of Australia \& New Zealand (HSANZ) as a 2006 Young Investigator. This work was further supported with funding from the Multiple Myeloma Research Foundation, the Leukemia \& Lymphoma Society, the Ontario Institute for Cancer Research, and the Ministry of Research and Innovation, Province of Ontario, Canada.

Received for publication October 4, 2007, and accepted in revised form March 5, 2008.

Address correspondence to: A. Keith Stewart, Mayo Clinic, Collaborative Research Building 3-001, 13400 E. Shea Boulevard, Scottsdale, Arizona 85259, USA. Phone: (480) 301-4411; Fax: (480) 301-8387; E-mail: stewart.keith@mayo.edu.
1. [No authors listed]. 2004. Cancer facts and figures 2004. American Cancer Society. Atlanta, Georgia, USA. 58 pP.

2. Bergsagel, P.L., et al. 2005. Cyclin D dysregulation: an early and unifying pathogenic event in multiple myeloma. Blood. 106:296-303.

3. Koziczak, M., Holbro, T., and Hynes, N.E. 2004. Blocking of FGFR signaling inhibits breast cancer cell proliferation through downregulation of D-type cyclins. Oncogene. 23:3501-3508.

4. Kozar, K., et al. 2004. Mouse development and cell proliferation in the absence of D-cyclins. Cell. 118:477-491.

5. Malumbres, M., et al. 2004. Mammalian cells cycle without the D-type cyclin-dependent kinases Cdk4 and Cdk6. Cell. 118:493-504.

6. Kozar, K., and Sicinski, P. 2005. Cell cycle progression without cyclin D-CDK4 and cyclin D-CDK6 complexes. Cell Cycle. 4:388-391.

7. Lahti, J.M., Li, H., and Kidd, V.J. 1997. Elimination of cyclin D1 in vertebrate cells leads to an altered cell cycle phenotype, which is rescued by overexpression of murine cyclins D1, D2, or D3 but not by a mutant cyclin D1. J. Biol. Chem. 272:10859-10869.

8. Carthon, B.C., et al. 2005. Genetic replacement of cyclin D1 function in mouse development by cyclin D2. Mol. Cell. Biol. 25:1081-1088.

9. Ciemerych, M.A., et al. 2002. Development of mice expressing a single D-type cyclin. Genes Dev. 16:3277-3289.

10. Sicinski, P., et al. 1996. Cyclin D2 is an FSH-responsive gene involved in gonadal cell proliferation and oncogenesis. Nature. 384:470-474.

11. Hurt, E.M., et al. 2004. Overexpression of c-maf is a frequent oncogenic event in multiple myeloma that promotes proliferation and pathological interactions with bone marrow stroma. Cancer Cell. 5:191-199.

12. Mao, X., et al. 2007. A chemical biology screen identifies glucocorticoids that regulate c-maf expression by increasing its proteasomal degradation through up-regulation of ubiquitin. Blood. 110:4047-4054.

13. National Toxicology Program. 1986. NTP Toxicology and Carcinogenesis Studies of C.I. Basic Red 9 Monohydrochloride (Pararosaniline) (CAS 569-619) In F344/N Rats and B6C3F1 Mice (Feed Studies). Natl. Toxicol. Program Tech. Rep. Ser. 285:1-228.

14. Au, W., Butler, M.A., Bloom, S.E., and Matney, T.S.
1979. Further study of the genetic toxicity of gentian violet. Mutat. Res. 66:103-112.

15. Hennequin, C., Giocanti, N., Balosso, J., and Favaudon, V. 1994. Interaction of ionizing radiation with the topoisomerase I poison camptothecin in growing V-79 and HeLa cells. Cancer Res. 54:1720-1728.

16. Shao, R.G., et al. 1997. Abrogation of an S-phase checkpoint and potentiation of camptothecin cytotoxicity by 7-hydroxystaurosporine (UCN-01) in human cancer cell lines, possibly influenced by p53 function. Cancer Res. 57:4029-4035.

17. Pandey, M.K., et al. 2007. Gambogic acid, a novel ligand for transferrin receptor, potentiates TNFinduced apoptosis through modulation of the nuclear factor-\{kappa\}B signaling pathway. Blood. 110:3517-3525.

18. Yore, M.M., Liby, K.T., Honda, T., Gribble, G.W., and Sporn, M.B. 2006. The synthetic triterpenoid 1-[2-cyano-3,12-dioxooleana-1,9(11)-dien-28oyl]imidazole blocks nuclear factor-kappaB activation through direct inhibition of IkappaB kinase beta. Mol. Cancer Ther. 5:3232-3239.

19. Werner, T., Motyka, V., Strnad, M., and Schmulling, T. 2001. Regulation of plant growth by cytokinin. Proc. Natl. Acad. Sci. U. S. A. 98:10487-10492.

20. Gray, W.M. 2004. Hormonal regulation of plant growth and development. PLoS Biol. 2:e311.

21. Riou-Khamlichi, C., Huntley, R., Jacqmard, A., and Murray, J.A. 1999. Cytokinin activation of Arabidopsis cell division through a D-type cyclin. Science. 283:1541-1544.

22. Guo, Y., Harwalkar, J., Stacey, D.W., and Hitomi, M. 2005. Destabilization of cyclin D1 message plays a critical role in cell cycle exit upon mitogen withdrawal. Oncogene. 24:1032-1042.

23. Jourdan, M., et al. 1998. The myeloma cell antigen syndecan-1 is lost by apoptotic myeloma cells. Br.J. Haematol. 100:637-646.

24. Muniz, L.C., Yehia, G., Memin, E., Ratnakar, P.V., and Molina, C.A. 2006. Transcriptional Regulation of Cyclin D2 by the PKA Pathway and Inducible cAMP Early Repressor (ICER) in Granulosa Cells. Biol. Reprod. 75:279-288.

25. Silverstein, A.M., Barrow, C.A., Davis, A.J., and Mumby, M.C. 2002. Actions of PP2A on the MAP kinase pathway and apoptosis are mediated by dis- tinct regulatory subunits. Proc. Natl. Acad. Sci. U. S. A. 99:4221-4226.

26. Krieger, S., Grunau, C., Sabbah, M., and Sola, B. 2005. Cyclin D1 gene activation in human myeloma cells is independent of DNA hypomethylation or histone hyperacetylation. Exp. Hematol. 33:652-659.

27. Muto, A., et al. 2002. Activation of Maf/AP-1 repressor Bach2 by oxidative stress promotes apoptosis and its interaction with promyelocytic leukemia nuclear bodies. J. Biol. Chem. 277:20724-20733.

28. Muto, A., et al. 1998. Identification of Bach2 as a B-cell-specific partner for small maf proteins that negatively regulate the immunoglobulin heavy chain gene 3' enhancer. EMBOJ. 17:5734-5743.

29. White, P.C., et al. 2006. Regulation of cyclin D2 and the cyclin D2 promoter by protein kinase A and CREB in lymphocytes. Oncogene. 25:2170-2180.

30. Razavi, R., Ramos, J.C., Yehia, G., Schlotter, F., and Molina, C.A. 1998. ICER-IIgamma is a tumor suppressor that mediates the antiproliferative activity of cAMP. Oncogene. 17:3015-3019.

31. Molina, C.A., Foulkes, N.S., Lalli, E., and SassoneCorsi, P. 1993. Inducibility and negative autoregulation of CREM: an alternative promoter directs the expression of ICER, an early response repressor. Cell. 75:875-886.

32. Laoide, B.M., Foulkes, N.S., Schlotter, F., and Sassone-Corsi, P. 1993. The functional versatility of CREM is determined by its modular structure. EMBO J. 12:1179-1191.

33. Stehle, J.H., et al. 1993. Adrenergic signals direct rhythmic expression of transcriptional repressor CREM in the pineal gland. Nature. 365:314-320.

34. Herber, B., Truss, M., Beato, M., and Muller, R. 1994. Inducible regulatory elements in the human cyclin D1 promoter. Oncogene. 9:1295-1304.

35. Moriuchi, A., et al. 2003. A CRE and the region occupied by a protein induced by growth factors contribute to up-regulation of cyclin D1 expression in hepatocytes. Biochem. Biophys. Res. Commun. 300:415-421.

36. Kim, M.J., et al. 2006. Exendin-4 induction of cyclin D1 expression in INS-1 beta-cells: involvement of cAMP-responsive element. J. Endocrinol. 188:623-633.

37. Cooper, A.B., et al. 2006. A unique function for cyclin D3 in early B cell development. Nat. Immunol. 
7:489-497.

38. Griffaut, B., Bos, R., Maurizis, J.C., Madelmont, J.C. and Ledoigt, G. 2004. Cytotoxic effects of kinetin riboside on mouse, human and plant tumour cells. Int. J. Biol. Macromol. 34:271-275.

39. Honma, Y., and Ishii, Y. 2002. Differentiation of human myeloid leukemia cells by plant redifferentiation-inducing hormones. Leuk. Lymphoma. 43:1729-1735.

40. Ishii, Y., Hori, Y., Sakai, S., and Honma, Y. 2002. Control of differentiation and apoptosis of human myeloid leukemia cells by cytokinins and cytokinin nucleosides, plant redifferentiation-inducing hormones. Cell Growth Differ. 13:19-26.

41. Sakane-Ishikawa, E., et al. 2005. Prognostic significance of BACH2 expression in diffuse large B-cell lymphoma: a study of the Osaka Lymphoma Study Group. J. Clin. Oncol. 23:8012-8017.

42. Muto, A., et al. 2004. The transcriptional programme of antibody class switching involves the repressor Bach2. Nature. 429:566-571.

43. Kamio, T., et al. 2003. B-cell-specific transcription factor BACH2 modifies the cytotoxic effects of anticancer drugs. Blood. 102:3317-3322.

44. Ge, L., et al. 2005. Identification of kinetin and kinetin riboside in coconut (Cocos nucifera L.) water using a combined approach of liquid chromatography-tandem mass spectrometry, high performance liquid chromatography and capillary electrophoresis. J. Chromatogr. B. Analyt. Technol. Biomed. Life Sci. 829:26-34.

45. Barciszewski, J., Mielcarek, M., Stobiecki, M., Siboska, G., and Clark, B.F. 2000. Identification of 6-furfuryladenine (kinetin) in human urine. Biochem. Biophys. Res. Commun. 279:69-73.

46. Epstein, H.A. 2004. Kinerase: the science behind the technology. Skinmed. 3:339.

47. Glaser, D.A., and Rogers, C. 2001. Topical and systemic therapies for the aging face. Facial Plast. Surg. Clin. North Am. 9:189-196.

48. Kligman, D. 2000. Cosmeceuticals. Dermatol. Clin 18:609-615.

49. Root, D.E., Hacohen, N., Hahn, W.C., Lander, E.S., and Sabatini, D.M. 2006. Genome-scale loss-offunction screening with a lentiviral RNAi library. Nat. Methods. 3:715-719.
50. Naldini, L., et al. 1996. In vivo gene delivery and stable transduction of nondividing cells by a lentiviral vector. Science. 272:263-267.

51. Brooks, A.R., Shiffman, D., Chan, C.S., Brooks, E.E., and Milner, P.G. 1996. Functional analysis of the human cyclin D2 and cyclin D3 promoters. J. Biol. Chem. 271:9090-9099.

52. Krajewski, S., Zapata, J.M., and Reed, J.C. 1996. Detection of multiple antigens on Western blots. Anal. Biochem. 236:221-228.

53. Keats, J.J., et al. 2007. Promiscuous mutations activate the noncanonical NF-kappaB pathway in multiple myeloma. Cancer Cell. 12:131-144.

54. Shi, C.X., Graham, F.L., and Hitt, M.M. 2006. A convenient plasmid system for construction of helper-dependent adenoviral vectors and its application for analysis of the breast-cancer-specific mammaglobin promoter. J. Gene Med. 8:442-451.

55. Birney, E., et al. 2006. Ensembl 2006. Nucleic Acids Res. 34:D556-D561.

56. Ashurst, J.L., et al. 2005. The Vertebrate Genome Annotation (Vega) database. Nucleic Acids Res. 33:D459-D465. 